\title{
THE RELAX CODES FOR LINEAR MINIMUM COST NETWORK FLOW PROBLEMS *
}

\author{
Dimitri P. BERTSEKAS and Paul TSENG
}

Laboratory for Information and Decision Systems and the Operations Research Center, Massachusetts Institute of Technology, Cambridge, Massachusetts 02139, USA

\begin{abstract}
We describe a relaxation algorithm $[1,2]$ for solving the classical minimum cost network flow problem. Our implementation is compared with mature state-of-the-art primal simplex and primal-dual codes and is found to be several times faster on all types of randomly generated network flow problems. Furthermore, the speed-up factor increases with problem dimension. The codes, called RELAX-II and RELAXT-II, have a facility for efficient reoptimization and sensitivity analysis, and are in the public domain.
\end{abstract}

\section{Introduction}

Consider a directed graph with a set of nodes $\mathcal{N}$ and a set of arcs $\mathcal{A}$. Each arc $(i, j)$ has associated with it an integer $a_{i j}$ referred to as the cost of $(i, j)$. We denote by $f_{i j}$ the flow of the arc $(i, j)$ and consider the classical minimum cost flow problem

$$
\begin{array}{lll}
\text { minimize } & \sum_{(i, j) \in S d} a_{i j} f_{i j} & \text { (MCF) } \\
\text { subject to } & \sum_{\substack{m \\
(m, i) \in \mathscr{A}}} f_{m i}-\sum_{\substack{m \\
(i, m) \in \mathscr{A}}} f_{i m}=0, \forall i \in \mathcal{N} & \text { (conservation of flow) } \\
& \ell_{i j} \leqslant f_{i j} \leqslant c_{i j}, \forall(i, j) \in \mathscr{A} & \text { (capacity constraint) }
\end{array}
$$

where $\ell_{i j}$ and $c_{i j}$ are given integers. We assume throughout that there exists at least one feasible solution of (MCF). We formulate a dual problem to (MCF).

We associate a Lagrange multiplier $p_{i}$ (referred to as the price of node $i$ ) with the $i$ th conservation of flow constraint (1). By denoting by $f$ and $p$ the vectors with elements $f_{i j},(i, j) \in \mathscr{A}$ and $p_{i}, i \in \mathcal{N}$, respectively, we can write the corresponding Lagrangian function

*This work has been supported by the National Science Foundation under Grant NSF-ECS8217668 .

(c) J.C. Baltzer AG, Scientific Publishing Company 


$$
L(f, p)=\sum_{(i, j) \in \mathcal{A}}\left(a_{i j}+p_{j}-p_{i}\right) f_{i j} .
$$

The dual problem is

$$
\begin{aligned}
& \text { maximize } q(p) \\
& \text { subject to no constraints on } p,
\end{aligned}
$$

where the dual functional $q$ is given by

$$
\begin{aligned}
q(p) & =\min _{\ell_{i j} \leqslant f_{i j} \leqslant c_{i j}} L(f, p) \\
& =\sum_{(i, j) \in \mathscr{A}} \min _{l_{i j} \leqslant f_{i j} \leqslant c_{i j}}\left\{\left(a_{i j}+p_{j}-p_{i}\right) f_{i j}\right\} \triangleq \sum_{(i, j) \in \mathscr{A}} q_{i j}\left(p_{i}-p_{j}\right) .
\end{aligned}
$$

The form of the dual arc cost functions $q_{i j}$ is shown in fig. 1 .
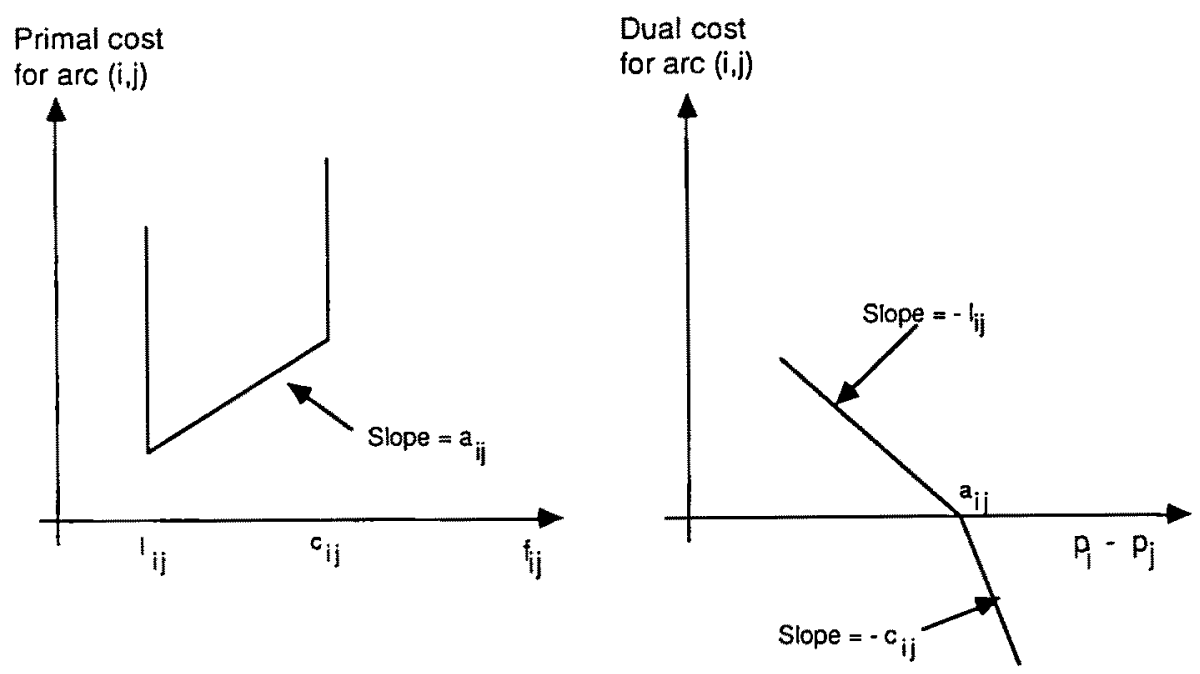

Fig. 1. Primal and dual costs for arc $(i, j)$.

Given any price vector $p$, we consider the corresponding tension vector $t$ having elements $t_{i j},(i, j) \in \mathscr{A}$ defined by

$$
t_{i j}=p_{i}-p_{j}, \forall(i, j) \in \mathbb{A} .
$$

Since the dual functional as well as subsequent definitions, optimality conditions and algorithms depend on the price vector $p$ only through the corresponding tension vector $t$, we will often make no distinction between $p$ and $t$ in what follows. 
For any price vector $p$, we say that an $\operatorname{arc}(i, j)$ is:

Inactive if $t_{i j}<a_{i j}$

Balanced if $t_{i j}=a_{i j}$

Active if $t_{i j}>a_{i j}$.

For any flow vector $f$, the scalar

$$
d_{i}=\sum_{m} f_{i m}-\sum_{m} f_{m i} \forall i \in \mathcal{N}
$$

will be referred to as the deficit of node $i$. It represents the difference of total flow exported and total flow imported by the node.

The optimality conditions in connection with $(\mathrm{MCF})$ and its dual given by (3) and (4) state that $(f, p)$ is a primal and dual optimal solution pair if and only if

$$
\begin{aligned}
& f_{i j}=\ell_{i j} \quad \text { for all inactive arcs }(i, j) \\
& \ell_{i j} \leqslant f_{i j} \leqslant c_{i j} \quad \text { for all balanced arcs }(i, j) \\
& f_{i j}=c_{i j} \quad \text { for all active arcs }(i, j) \\
& d_{i}=0 \quad \text { for all nodes } i \text {. }
\end{aligned}
$$

Relations (10)-(12) are known as the complementary slackness conditions.

Our approach is based on iterative ascent of the dual functional. The price vector $p$ is updated while simultaneously maintaining a flow vector $f$ satisfying complementary slackness with $p$. The algorithms proposed terminate when $f$ satisfies primal feasibility (deficit of each node equals zero). The main feature of the algorithms, which distinguishes them from classical primal-dual methods, is that the choice of ascent directions is very simple. At a given price vector $p$, a node $i$ with nonzero deficit is chosen, and an ascent is attempted along the coordinate $p_{i}$. If such an ascent is not possible and a reduction of the total absolute deficit $\Sigma_{m}\left|d_{m}\right|$ cannot be effected through flow augmentation, an adjacent node of $i$, say $i_{1}$, is chosen and an ascent is attempted along the sum of the coordinate vectors corresponding to $i$ and $i_{1}$. If such an ascent is not possible, and flow augmentation is not possible either, an adjacent node of either $i$ or $i_{1}$ is chosen and the process is continued. In practice, most of the ascent directions are single coordinate directions, leading to the interpretation of the algorithms as coordinate ascent or relaxation methods. This is an important characteristic, and a key factor in the algorithms' efficiency. We have found through experiment that, for ordinary networks, the ascent directions used 
by our algorithms lead to comparable improvement per iteration as the direction of maximal rate of ascent (the one used by the classical primal-dual method), but are computed with considerably less overhead.

In the next section, we characterize the ascent directions used in the algorithms. In sect. 3, we describe our relaxation methods. In sect. 4, we describe the codes and give results of computational experimentation.

\section{Characterization of ascent directions}

Each ascent direction used by the algorithm is associated with a connected strict subset $S$ of $\mathcal{N}$, and has the form $v=\left\{v_{i j} \mid(i, j) \in \mathscr{A}\right\}$, where

$$
v_{i j}=\left\{\begin{aligned}
1 & \text { if } i \notin S, j \in S \\
-1 & \text { if } i \in S, j \notin S \\
0 & \text { otherwise. }
\end{aligned}\right.
$$

Changing any tension vector $t$ in the direction $v$ of (14) corresponds to decreasing the prices of all nodes in $S$ by an equal amount while leaving the prices of all other nodes unchanged. It is seen from (4) that the directional derivative at $t$ of the dual cost in the direction $v$ is $C(v, t)$, where

$$
\begin{aligned}
C(v, t) & =\sum_{(i, j) \in \mathscr{A}} \lim _{\alpha \rightarrow 0^{+}} \frac{q_{i j}\left(t_{i j}+\alpha v_{i j}\right)-q_{i j}\left(t_{i j}\right)}{\alpha} \\
& =\sum_{(i, j) \in \mathscr{A}} e_{i j}\left(v_{i j}, t_{i j}\right)
\end{aligned}
$$

and

$$
e_{i j}\left(v_{i j}, t_{i j}\right)= \begin{cases}-v_{i j} \ell_{i j} & \text { if } \quad(i, j) \text { is inactive or if }(i, j) \\
& \text { is balanced and } v_{i j} \leqslant 0 \\
-v_{i j} c_{i j} & \text { if } \quad \begin{array}{l}
(i, j) \text { is active or if }(i, j) \\
\text { is balanced and } v_{i j} \geqslant 0 .
\end{array}\end{cases}
$$

Note that $C(v, t)$ is the difference of outflow and inflow across $S$ when the flows of inactive and active arcs are set at their lower and upper bounds, respectively, while the flow of each balanced arc incident to $S$ is set to its lower or upper bound depending on whether the arc is going out of $S$ or coming into $S$, respectively. We have the following proposition. 


\section{PROPOSITION 1}

For every non-empty strict subset $S$ of $\mathcal{N}$ and every tension vector $t$, there holds

$$
w(t+\gamma v)=w(t)+\gamma C(v, t), \quad \forall \gamma \in[0, \delta),
$$

where $w(\cdot)$ is the dual cost as a function of $t$

$$
w(t)=\sum_{(i, j)} q_{i j}\left(t_{i j}\right)
$$

Here, $v$ is given by (14) and $\delta$ is given by

$$
\begin{array}{r}
\delta=\inf \left\{\left\{t_{i m}-a_{i m} \mid i \in S, m \notin S,(i, m): \text { active }\right\},\right. \\
\left.\left\{a_{m i}-t_{m i} \mid i \in S, m \notin S,(m, i): \text { inactive }\right\}\right\} .
\end{array}
$$

(We use the convention $\delta=+\infty$ if the set over which the infimum above is taken is empty.)

Proof

It was seen [cf. (15)] that the rate of change of the dual cost $w$ at $t$ along $v$ is $C(v, t)$. Since $w$ is piecewise linear, the actual change of $w$ along the direction $v$ is linear in the stepwise $\gamma$ up to the point where $\gamma$ becomes large enough so that the pair $[w(t+\gamma v), t+\gamma v]$ meets a new face of the graph of $w$. This value of $\gamma$ is the one for which a new arc incident to $S$ becomes balanced and it equals the scalar $\delta$ of (19).

Q.E.D.

\section{The relaxation method}

The relaxation algorithm maintains complementary slackness at all times. At each iteration, it starts from a single node with nonzero deficit and checks whether changing its price can improve the value of the dual cost. If not, it gradually builds up, via a labeling procedure, either a flow augmenting path or a cutset associated with a direction of ascent. The main difference from the classical primal-dual method is that instead of continuing the labeling process until a maximal set of nodes is labeled, we stop at the first possible direction of ascent - frequently the direction associated with just the starting node. 


\section{TYPICAL RELAXATION ITERATION FOR AN ORDINARY NETWORK}

At the beginning of each iteration, we have a pair $(f, t)$ satisfying complementary slackness. The iteration determines a new pair $(f, t)$ satisfying complementary slackness by means of the following process.

Step 1: Choose a node $s$ with $d_{s}>0$. (The iteration can be started also from a node $s$ with $d_{s}<0-$ the steps are similar.) If no such node can be found, terminate the algorithm. Else give the label " 0 " to $s$, set $S=\emptyset$, and go to step 2 . Nodes in $S$ are said to be scanned.

Step 2: Choose a labeled but unscanned node $k$, set $S=S \cup\{k\}$, and go to step 3 .

Step 3: Scan the label of the node $k$ as follows: Give the label " $k$ " to all unlabeled nodes $m$ such that $(m, k)$ is balanced and $f_{m k}<c_{m k}$, and to all unlabeled $m$ such that $(k, m)$ is balanced and $\ell_{k m}<f_{k m}$. If $v$ is the vector corresponding to $S$ as in (14) and

$$
C(v, t)>0
$$

go to step 5 . Else if for any of the nodes $m$ labeled from $k$ we have $d_{m}<0$, go to step 4. Else go to step 2.

Step 4 (flow augmentation): A directed path $P$ has been found that begins at the starting node $s$ and ends at the node $m$ with $d_{m}<0$ identified in step 3 . The path is constructed by tracing labels backwards starting from $m$, and consists of balanced arcs such that we have $\ell_{k n}<f_{k n}$ for all $(k, n) \in P^{+}$and $f_{k n}<c_{k n}$ for all $(k, n) \in P^{-}$, where

$$
\begin{aligned}
& P^{+}=\{(k, n) \in P \mid(k, n) \text { is oriented in the direction from } s \text { to } m\} \\
& P^{-}=\{(k, n) \in P \mid(k, n) \text { is oriented in the direction from } m \text { to } s\}
\end{aligned}
$$

Let

$$
\epsilon=\min \left\{d_{s^{\prime}}-d_{m},\left\{f_{k n}-\ell_{k n} \mid(k, n) \in P^{+}\right\},\left\{c_{k n}-f_{k n} \mid(k, n) \in P^{-}\right\}\right\} .
$$

Decrease by $\epsilon$ the flows of all arcs $(k, n) \in P^{+}$, increase by $\epsilon$ the flows of all arcs $(k, n) \in P^{-}$, and go to the next iteration. 
Step 5 (price adjustment): Let

$$
\begin{aligned}
& \delta=\min \left\{t_{k m}-a_{k m} \mid k \in S, m \notin S,(k, m): \text { active }\right\}, \\
&\left\{a_{m k}-t_{m k} \mid k \in S, m \notin S,(m, k): \text { inactive }\right\},
\end{aligned}
$$

where $S$ is the set of scanned nodes constructed in step 2 . Set

$$
\begin{gathered}
f_{k m}:=\ell_{k m}, \forall \text { balanced } \operatorname{arcs}(k, m) \text { with } k \in S, m \in L, m \notin S \\
f_{m k}:=c_{m k}, \forall \text { balanced } \operatorname{arcs}(m, k) \text { with } k \in S, m \in L, m \notin S,
\end{gathered}
$$

where $L$ is the set of labeled nodes. Set

$$
t_{k m}:=\left\{\begin{array}{llr}
t_{k m}+\delta & \text { if } & k \notin S, m \in S \\
t_{k m}-\delta & \text { if } \quad k \in S, m \notin S \\
t_{k m} & \text { otherwise. }
\end{array}\right.
$$

Go to the next iteration.

The relaxation iteration terminates with either a flow augmentation (via step 4) or with a dual cost improvement (via step 5). In order for the procedure to be well defined, however, we must show that whenever we return to step 2 from step 3, there is still some labeled node which is unscanned. Indeed, when all labeled nodes are scanned (i.e. the set $S$ coincides with the labeled set), there is no balanced arc $(m, k)$ such that $m \notin S, k \in S$ and $f_{m k}<c_{m k}$ or a balanced arc $(k, m)$ such that $k \in S, m \notin S$ and $f_{k m}>\ell_{k m}$. It follows from the definition (15), (16) [see also the following equation (25)] that

$$
C(v, t)=\sum_{k \in S} d_{k}
$$

Under the above circumstances, all nodes in $S$ have nonnegative deficit and at least one node in $S$ (the starting node $s$ ) has strictly positive deficit. Therefore, $C(v, t)>0$ and it follows that the procedure switches from step 3 to step 5 rather than switch back to step 2 .

If $a_{i j}, \ell_{i j}$, and $c_{i j}$ are integer for all $(i, j) \in \mathscr{A}$ and the starting $t$ is integer, then $\delta$ as given by (24) will also be a positive integer and the dual cost is increased by an integer amount each time step 5 is executed. Each time a flow augmentation takes place via step 4 , the dual cost remains unchanged. If the starting $f$ is integer, all 
successive $f$ will be integer, so the amount of flow augmentation $\epsilon$ in step 4 will be a positive integer. Therefore, there can be only a finite number of flow augmentations between successive reductions of the dual cost. It follows that the algorithm will finitely terminate at an integer optimal pair $(f, t)$ if the starting pair $(f, t)$ is integer.

It can be seen that the relaxation iteration involves a comparable amount of computation per node scanned as the usual primal-dual method [3]. The only additional computation involves maintaining the quantity $C(v, t)$, but it can be seen that this can be computed incrementally in step 3 rather than recomputed each time the set $S$ is enlarged in step 2 . As a result, this additional computation is insignificant. To compute $C(v, t)$ incrementally in the context of the algorithm, it is helpful to use the identity

$$
C(v, t)=\sum_{i \in S} d_{i}-\sum_{\substack{(i, j): \text { balanced } \\ i \in S, i \notin S}}\left(f_{i j}-l_{i j}\right)-\sum_{\substack{(i, j): \text { balanced } \\ i \notin S, j \in S}}\left(c_{i j}-f_{i j}\right) .
$$

We note that a similar iteration can be constructed starting from a node with negative deficit. Here, the set $S$ consists of nodes with nonpositive deficit, and in step 5 , the prices of the nodes in $S$ are increased rather than decreased. The straightforward details are left to the reader. Computational experience suggests that termination is typically accelerated when ascent iterations are initiated from nodes with negative as well as positive deficit.

\section{LINE SEARCH}

The stepsize $\delta$ of (24) corresponds to the first break point of the (piecewise linear) dual functional along the ascent direction. It is possible to instead use an optimal stepsize that maximizes the dual functional along the ascent direction. Such a stepsize can be calculated quite efficiently by testing the sign of the directional derivative of the dual cost at successive break points along the ascent direction. Computational experimentation showed that this type of line search is beneficial, and was implemented in the relaxation codes.

\section{SINGLE NODE ITERATIONS}

The case where the relaxation iteration scans a single node (the starting node $s$ having positive deficit $d_{s}$ ), finds the corresponding direction $v_{s}$ to be an ascent direction, i.e.

$$
C\left(v_{s}, t\right)=d_{s}-\sum_{(s, m): \text { balanced }}\left(f_{s m}-\ell_{s m}\right)-\sum_{(m, s): \text { balanced }}\left(c_{m s}-f_{m s}\right)>0,
$$

reduces the price $p_{s}$ (perhaps repeatedly via the line search mentioned earlier) and terminates is particularly important for the conceptual understanding of the algorithm. 

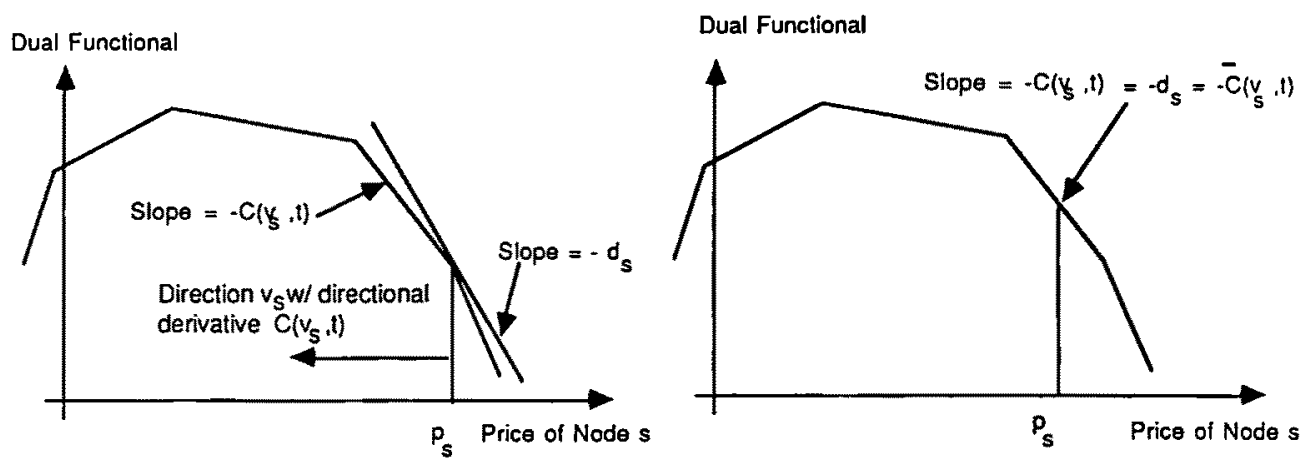

CASES WHERE A SINGLE NODE ITERATION IS POSSIBLE

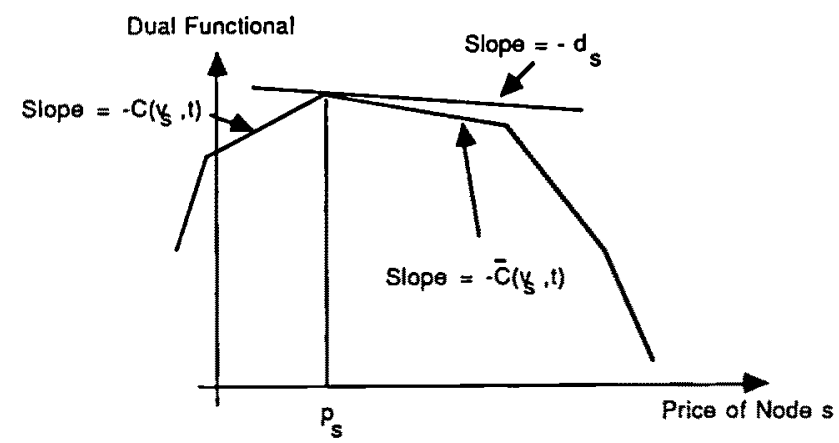

CASE WHERE A SINGLE NODE ITERATION IS NOT POSSIBLE

Fig. 2. Illustration of dual functional and its directional derivatives along the price coordinate $p_{s}$. Break points correspond to values of $p_{s}$ where one or more arcs incident to node $s$ are balanced.

We believe that much of the success of the algorithm is owed to the relatively large number of single node iterations for many classes of problems.

When only the price of a single node $s$ is changed, the absolute value of the deficit of $s$ is decreased at the expense of possibly increasing the absolute value of the deficit of its neighboring nodes. This is reminiscent of relaxation methods where a change of a single variable is effected with the purpose of satisfying a single constraint at the expense of violating others.

A dual viewpoint, reminiscent of coordinate ascent methods, is that a single (the $s$ th) coordinate direction is chosen and a line search is performed along this direction. Figure 2 shows the form of the dual function along the direction of the coordinate $p_{s}$ for a node with

$$
d_{s}>0 \text {. }
$$


The left-hand slope at $p_{s}$ is

$$
-C\left(v_{s}, t\right)
$$

while the right-hand slope is

$$
\begin{aligned}
& -\bar{C}\left(v_{s}, t\right)=-\sum_{\substack{(s, m) \in s d \\
(s, m): \text { active } \\
\text { or balanced }}} c_{s m}-\sum_{\substack{(s, m) \in \mathscr{A} \\
(s, m): \text { inactive }}} l_{s m} \\
& +\sum_{\substack{(m, s) \in \mathbb{A} \\
(m, s): \text { active }}} c_{m s}+\sum_{\substack{(m, s) \in A \\
(m, s): \text { inactive } \\
\text { or balanced }}} l_{m s} .
\end{aligned}
$$

We have

$$
-\bar{C}\left(v_{s}, t\right) \leqslant-d_{s} \leqslant-C\left(v_{s}, t\right)
$$

so $-d_{s}$ is a subgradient of the dual functional at $p_{s}$ in the $s$ th coordinate direction.

A single node iteration will be possible if and only if the right-hand slope is negative or equivalently

$$
C\left(v_{s}, t\right)>0
$$

This will always be true if we are not at a corner and hence equality holds throughout in (27). However, if the dual cost is nondifferentiable at $p_{s}$ along the sth coordinate, it may happen that (see fig. 2)

$$
-\bar{C}\left(v_{s}, t\right) \leqslant-d_{s}<0 \leqslant-C\left(v_{s}, t\right)
$$

in which case the single node iteration fails to make progress and we must resort to scanning more than one node.

Figure 3 illustrates a single node iteration for the case where $d_{s}>0$. It is seen that the break points of the dual functional along the coordinate $p_{s}$ are the values of $p_{s}$ for which one or more arcs incident to node $s$ are balanced. The single node iteration shown starts with arcs $(1, s)$ and $(3, s)$ inactive, and arcs $(s, 2)$ and $(s, 4)$ active. To reduce $p_{s}$ beyond the first break point $p_{4}+a_{s 4}$. the flow of arc $(s, 4)$ must be pulled back from $f_{s 4}=30$ to $f_{s 4}=0$. At the level $p_{3}-a_{3 s}$, the dual cost is maximized because if the flow of arc $(3, s)$ is set to the lower bound of zero, the deficit $d_{s}$ switches from positive $(+10)$ to negative $(-10)$. Figure 4 illustrates a single node iteration for the same node when $d_{s}<0$. The difference to the case $d_{s}>0$ is that 


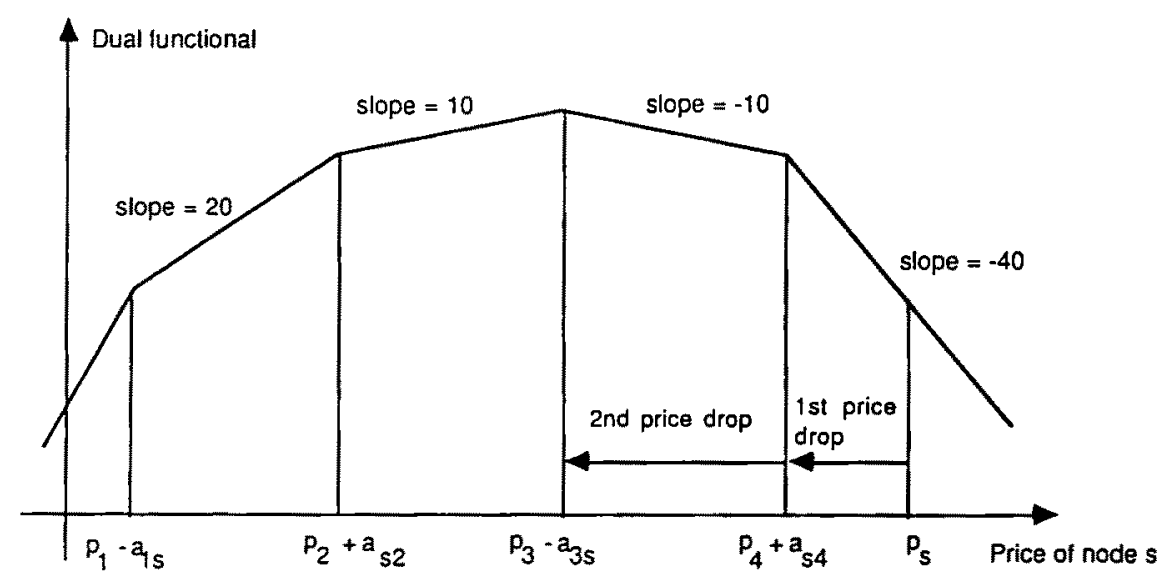

(a)

Price level

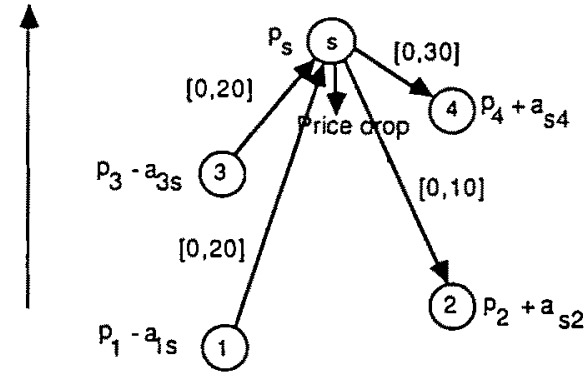

(b)

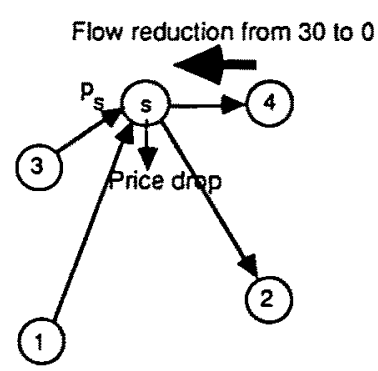

(c)

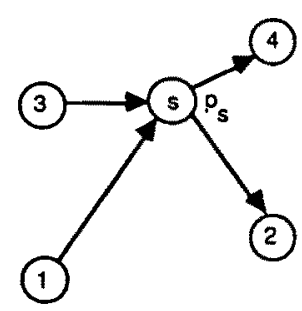

(d)

Fig. 3. Illustration of an iteration involving a single node $s$ with four adjacent arcs $(1, s),(3, s),(s, 2),(s, 4)$ with feasible arc flow ranges $[1,20],[0,20],[0,10]$, $[0,30]$, respectively. (a) Form of the dual functional along $p_{s}$ for given values of $p_{1}, p_{2}, p_{3}$, and $p_{4}$. The break points correspond to the levels of $p_{s}$ for which the corresponding arcs become balanced. (b) Illustration of a price drop of $p_{s}$ from a value higher than all break points to the break point at which arc $(s, 4)$ becomes balanced. (c) Price drop of $p_{s}$ to the break point at which arc $(3, s)$ becomes balanced. When this is done, arc $(s, 4)$ becomes inactive from balanced and its flow is reduced from 30 to 0 to maintain complementary slackness. (d) $p_{s}$ is now at the break point $p_{3}-a_{3 s}$ that maximizes the dual cost. Any further price drop makes arc $(3, s)$ active, increases its flow from 0 to 20 , and changes the sign of the deficit $d_{s}$ from positive $(+10)$ to negative $(-10)$. 


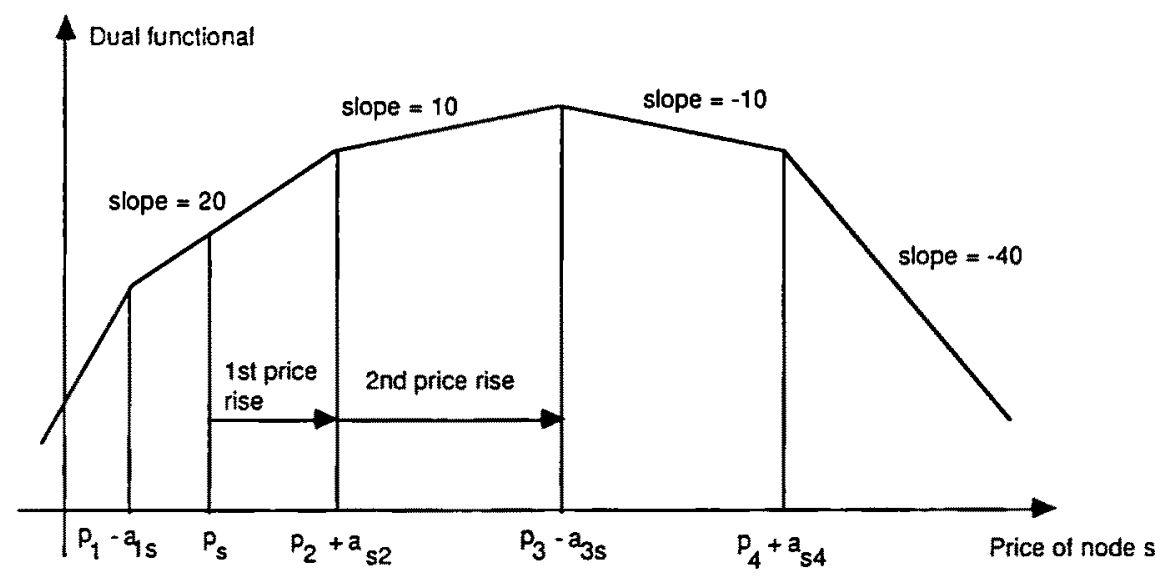

(a)

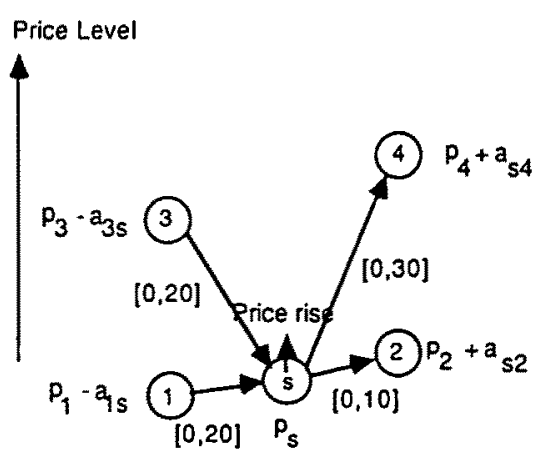

(b)

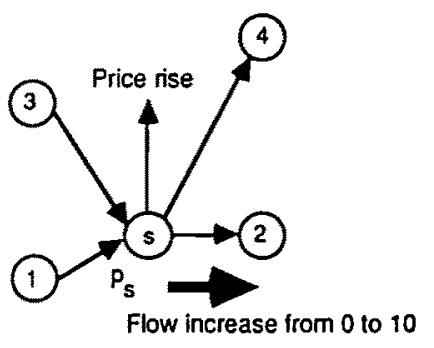

(c)

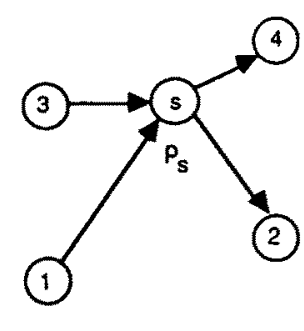

(d)

Fig. 4. Illustration of a price rise involving the single node $s$ for the example of fig. 3. Here, the initial price $p_{s}$ lies between the two leftmost break points corresponding to the arcs $(1, s)$ and $(s, 2)$. Initially, $\operatorname{arcs}(1, s),(s, 2)$, and $(s, 4)$ are inactive, and arc $(3, s)$ is active. 
the price $p_{s}$ is increased, instead of decreased, and as $p_{s}$ moves beyond a break point, the flow of the corresponding balanced arc is pushed to the lower bound (for incoming arcs) and to the upper bound (for outgoing arcs), rather than pulled to the upper bound and lower bound, respectively.

\section{DEGENERATE ASCENT ITERATIONS}

If, for a given $t$, we can find a connected subset $S$ of $\mathcal{N}$ such that the corresponding vector $(u, v)$ satisfies

$$
C(v, t)=0 \text {, }
$$

then from proposition 1 we see that the dual cost remains constant as we start moving along the vector $v$.i.e.

$$
w(t+\gamma v)=w(t), \quad \forall \gamma \in[0, \delta),
$$

where $w, v$, and $\delta$ are given by (14), (18), (19). We refer to such incremental changes in $t$ as degenerate ascent iterations. If the ascent condition $C(v, t)>0$ [cf. (20)] is replaced by $C(v, t) \geqslant 0$, then we obtain an algorithm that produces at each iteration either a flow augmentation, or a strict dual cost improvement, or a degenerate ascent step. This algorithm has the same convergence properties as the one without degenerate steps under the following condition:

(C) For each degenerate ascent iteration, the starting node $s$ has positive deficit $d_{s}$, and at the end of the iteration, all nodes in the scanned set $S$ have nonnegative deficit.

We refer the reader to [1] for a proof of this fact. It can be easily seen that condition (C) always holds when the set $S$ consists of just the starting node $s$. For this reason, if the ascent iteration is modified so that a price adjustment at step 5 is made not only when $C(v, t)>0$ but also when $d_{s}>0, s=\{s\}$ and $C\left(v_{s}, t\right)=0$, the algorithm maintains its termination properties. This modification was implemented in the relaxation codes and can have an important beneficial effect for special classes of problems such as assignment and transportation problems. We have no clear explanation for this phenomenon. For the assignment problem, condition (C) is guaranteed to hold even if $S$ contains more than one node. The assignment algorithm of [4] makes extensive use of degenerate ascent steps.

\section{Code description and computational results}

The relaxation codes RELAX-II and RELAXT-II solve the problem 


$$
\begin{array}{lll}
\text { minimize } & \sum_{(i, j) \in \mathscr{A}} a_{i j} f_{i j} & \\
\text { subject to } & \sum_{(m, i) \in \mathscr{A}} f_{m i}-\sum_{(i, m) \in \mathscr{A}} f_{i m}=b_{i}, & \forall i \in \mathcal{N} \\
& 0 \leqslant f_{i j} \leqslant c_{i j}, & \forall(i, j) \in \mathscr{A} .
\end{array}
$$

This form has become standard in network codes as it does not require storage and use of the array of lower bounds $\left\{\ell_{i j}\right\}$. Instead, the smaller size array $\left\{b_{i}\right\}$ is stored and used. The problem (MCF) of sect. 1 can be reduced to the above form by making the transformation of variables $f_{i j}:=f_{i j}-l_{i j}$. The method for representing the problem is the linked list structure suggested by Aashtiani and Magnanti [5] and used in their KILTER code (see also Magnanti [6]). Briefly, during solution of the problem, we store for each arc its start and end node, its capacity, its reduced cost $\left(a_{i j}-t_{i j}\right)$, its flow $f_{i j}$, the next arc with the same start node, and the next arc with the same end node. An additional array of length equal to half the number of arcs is used for internal calculations. This array could be eliminated at the expense of a modest increase in computation time. The total storage of RELAX-II for arc length arrays is $7.5|\$ A|$. RELAXT-II is a code that is similar to RELAX-II but employs two additional arc length arrays that essentially store the set of all balanced arcs. This code, written with the assistance of Jon Eckstein, is faster than RELAX-II, but requires $9.5|\not|$ total storage for arc length arrays. There is additional storage needed for node length arrays, but this is relatively insignificant for all but extremely sparse problems. This compares unfavorably with primal simplex codes, which can be implemented with four arc length arrays.

The RELAX-II and RELAXT-II codes implement with minor variations the relaxation algorithm of sect. 3 . Line search and degenerate ascent steps are implemented as discussed in sect. 3 .

The codes assume no prior knowledge about the structure of the problem or the nature of the solution. Initial prices are set to zero and initial arc flows are set to zero or the upper bound, depending on whether the arc cost is nonnegative or negative, respectively. RELAX-II and RELAXT-II include a preprocessing phase (included in the CPU time reported) whereby arc capacities are reduced to as small a value as possible without changing optimal solutions of the problem. Thus, for transportation problems, the capacity of each arc is set at the minimum of the supply and demand at the start and end nodes of the arc. We found experimentally that this preprocessing can markedly improve the performance of relaxation methods, particularly for transportation problems. We do not fully understand the nature of this phenomenon, but it is apparently related to the fact that tight arc capacities tend to make the shape of the isocost surfaces of the dual functional more "round". Generally speaking, tight 
arc capacity bounds increase the frequency of single node iterations. This behavior is in sharp contrast with that of primal simplex, which benefits from loose arc capacity bounds (fewer extreme points to potentially search over), and appears to be one of the main reasons for the experimentally observed superiority of relaxation over primal simplex for heavily capacitated problems.

It is possible to reduce the memory requirements of the codes by ordering the arc list of the network by head node, i.e. the outgoing arcs of the first node are listed first, followed by the outgoing arcs of the second node, etc. (forward star representation). If this is done, one arc length array becomes unnecessary, thereby reducing the memory requirements of RELAX-II to 6.5 arc length arrays, and of RELAXT-II to 8.5 arc length arrays. The problem solution time remains essentially unaffected by this device, but the time needed to prepare (or alter) the problem data will be increased. The same technique can also be used to reduce the memory requirements of the primal simplex method to three arc length arrays.

We have compared RELAX-II and RELAXT-II under identical test conditions with the primal-dual code KILTER (Aashtiani and Magnanti [5]) and the primal simplex code RNET (Grigoriadis and Hsu [7]). It is generally recognized that the performance of RNET is representative of the best that can be achieved with presently available simplex network codes written in FORTRAN. For example, Kennington and Helgason in their 1980 book [8] (p. 255) compare RNET with their own primal simplex code NETFLO on the first 35 NETGEN benchmarks [9] and conclude that "RNET . . produced the shortest times that we have seen on these 35 test problems". Our computational results with these benchmarks are given in table 1 and show substantially faster computation times for the relaxation codes over both KILTER and RNET.

An important and intriguing property of RELAX-II and RELAXT-II is that their speedup factor over RNET apparently increases with the size of the problem. This can be seen by comparing the results for the small problems $1-35$ with the results for the larger problems $37-40$ of table 1 . The comparison shows an improvement in the speedup factor that is not spectacular, but is noticeable and consistent. Table 2 shows that for even larger problems, the speedup factor increases further with problem dimension, and reaches or exceeds an order of magnitude (see fig. 5). This is particularly true for assignment problems where, even for relatively small problems, the speedup factor is of the order of 20 or more.

We note that there was some difficulty in generating the transportation problems of this table with NETGEN. Many of the problems generated were infeasible because some node supplies and demands were coming out zero or negative. This was resolved by adding the same number (usually 10 ) to all source supplies and all sink demands, as noted in table 2. Note that the transportation problems of the table are divided into groups and each group has the same average degree per node ( 8 for problems $6-15$, and 20 for problems $16-20$ ). 
Table 1

Standard Benchmark Problems 1-40 of [9] obtained using NETGEN. All times are in secs on a VAX 11/750. All codes compiled by FORTRAN in OPTIMIZE mode under VMS version 3.7, and under VMS version 4.1, as indicated. All codes run on the same machine under identical conditions. Problem 36 could not be generated with our version of NETGEN

\begin{tabular}{|c|c|c|c|c|c|c|c|}
\hline $\begin{array}{l}\text { Problem } \\
\text { type }\end{array}$ & $\begin{array}{c}\text { Problem } \\
\text { no. }\end{array}$ & $\begin{array}{l}\text { No. of } \\
\text { nodes }\end{array}$ & $\begin{array}{l}\text { No. of } \\
\text { arcs }\end{array}$ & $\begin{array}{r}\text { RELAX-II } \\
\text { (VMS } 3.7 / \\
\text { VMS 4.1) }\end{array}$ & $\begin{array}{c}\text { RELAXT-II } \\
\text { (VMS 3.7/ } \\
\text { VMS 4.1) }\end{array}$ & $\begin{array}{l}\text { KILTER } \\
\text { VMS } 3.7\end{array}$ & $\begin{array}{c}\text { RNET } \\
\text { VMS } 3.7\end{array}$ \\
\hline \multirow{10}{*}{ 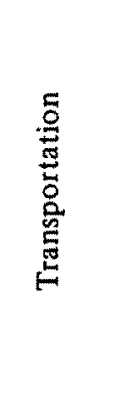 } & 1 & 200 & 1300 & $2.07 / 1.75$ & $1.47 / 1.22$ & 8.81 & 3.15 \\
\hline & 2 & 200 & 1500 & $2.12 / 1.76$ & $1.61 / 1.31$ & 9.04 & 3.72 \\
\hline & 3 & 200 & 2000 & $1.92 / 1.61$ & $1.80 / 1.50$ & 9.22 & 4.42 \\
\hline & 4 & 200 & 2200 & $2.52 / 2.12$ & $2.38 / 1.98$ & 10.45 & 4.98 \\
\hline & 5 & 200 & 2900 & $2.97 / 2.43$ & $2.53 / 2.05$ & 16.48 & 7.18 \\
\hline & 6 & 300 & 3150 & $4.37 / 3.66$ & $3.57 / 3.00$ & 25.08 & 9.43 \\
\hline & 7 & 300 & 4500 & $5.46 / 4.53$ & $3.83 / 3.17$ & 35.55 & 12.60 \\
\hline & 8 & 300 & 5155 & $5.39 / 4.46$ & $4.30 / 3.57$ & 46.30 & 15.31 \\
\hline & 9 & 300 & 6075 & $6.38 / 5.29$ & $5.15 / 4.30$ & 43.12 & 18.99 \\
\hline & 10 & 300 & 6300 & $4.12 / 3.50$ & $3.78 / 3.07$ & 47.80 & 16.44 \\
\hline \multicolumn{4}{|c|}{ Total (problems $1-10$ ) } & $37.32 / 31.11$ & $30.42 / 25.17$ & 251.85 & 96.22 \\
\hline \multirow{5}{*}{ 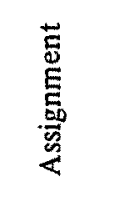 } & 11 & 400 & 1500 & $1.23 / 1.03$ & $1.35 / 1.08$ & 8.09 & 4.92 \\
\hline & 12 & 400 & 2250 & $1.38 / 1.16$ & $1.54 / 1.25$ & 10.76 & 6.43 \\
\hline & 13 & 400 & 3000 & $1.68 / 1.42$ & $1.87 / 1.54$ & 8.99 & 8.92 \\
\hline & 14 & 400 & 3750 & $2.43 / 2.07$ & $2.67 / 2.16$ & 14.52 & 9.90 \\
\hline & 15 & 400 & 4500 & $2.79 / 2.34$ & $3.04 / 2.46$ & 14.53 & 10.20 \\
\hline \multicolumn{4}{|c|}{ Total (problems $11-15$ ) } & $9.51 / 8.02$ & $10.47 / 8.49$ & 56.89 & 40.37 \\
\hline 尊 & 16 & 400 & 1306 & $2.79 / 2.40$ & $2.60 / 2.57$ & 13.57 & 2.76 \\
\hline 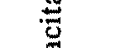 & 17 & 400 & 2443 & $2.67 / 2.29$ & $2.80 / 2.42$ & 16.89 & 3.42 \\
\hline 总 & 18 & 400 & 1306 & $2.56 / 2.20$ & $2.74 / 2.39$ & 13.05 & 2.56 \\
\hline & 19 & 400 & 2443 & $2.73 / 2.32$ & $2.83 / 2.41$ & 17.21 & 3.61 \\
\hline E & 20 & 400 & 1416 & $2.85 / 2.40$ & $2.66 / 2.29$ & 11.88 & 3.00 \\
\hline$=\frac{50}{9}$ & 21 & 400 & 2836 & $3.80 / 3.23$ & $3.77 / 3.23$ & 19.06 & 4.48 \\
\hline$\approx 0$ & 22 & 400 & 1416 & $2.56 / 2.18$ & $2.82 / 2.44$ & 12.14 & 2.86 \\
\hline$\ddot{\mathscr{E}}$ & 23 & 400 & 2836 & $4.91 / 4.24$ & $3.83 / 3.33$ & 19.65 & 4.58 \\
\hline 苞 & 24 & 400 & 1382 & $1.27 / 1.07$ & $1.47 / 1.27$ & 13.07 & 2.63 \\
\hline 䓢 & 25 & 400 & 2676 & $2.01 / 1.68$ & $2.13 / 1.87$ & 26.17 & 5.84 \\
\hline 总 & 26 & 400 & 1382 & $1.79 / 1.57$ & $1.60 / 1.41$ & 11.31 & 2.48 \\
\hline$\underline{\Xi}$ & 27 & 400 & 2676 & $2.15 / 1.84$ & $1.97 / 1.75$ & 18.88 & 3.62 \\
\hline \multicolumn{4}{|c|}{ Total (problems $16-27$ ) } & $32.09 / 27.42$ & $31.22 / 27.38$ & 192.88 & 41.94 \\
\hline
\end{tabular}


Table 1 (continued)

\begin{tabular}{|c|c|c|c|c|c|c|c|}
\hline $\begin{array}{l}\text { Problem } \\
\text { type }\end{array}$ & $\begin{array}{c}\text { Problem } \\
\text { no. }\end{array}$ & $\begin{array}{l}\text { No. of } \\
\text { nodes }\end{array}$ & $\begin{array}{l}\text { No. of } \\
\text { arcs }\end{array}$ & $\begin{array}{r}\text { RELAX-II } \\
\text { (VMS 3.7/ } \\
\text { VMS 4.1) }\end{array}$ & $\begin{array}{c}\text { RELAXT-II } \\
\text { (VMS 3.7/ } \\
\text { VMS 4.1) }\end{array}$ & $\begin{array}{l}\text { KILTER } \\
\text { VMS } 3.7\end{array}$ & $\begin{array}{c}\text { RNET } \\
\text { VMS } 3.7\end{array}$ \\
\hline \multirow{8}{*}{ 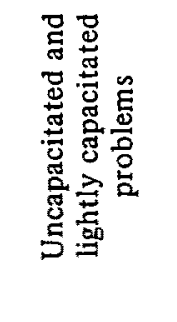 } & 28 & 1000 & 2900 & $4.90 / 4.10$ & $5.67 / 5.02$ & 29.77 & 8.60 \\
\hline & 29 & 1000 & 3400 & $5.57 / 4.76$ & $5.13 / 4.43$ & 32.36 & 12.01 \\
\hline & 30 & 1000 & 4400 & $7.31 / 6.47$ & $7.18 / 6.26$ & 42.21 & 11.12 \\
\hline & 31 & 1000 & 4800 & $5.76 / 4.95$ & $7.14 / 6.30$ & 39.11 & 10.45 \\
\hline & 32 & 1500 & 4342 & $8.20 / 7.07$ & $8.25 / 7.29$ & 69.28 & 18.04 \\
\hline & 33 & 1500 & 4385 & $10.39 / 8.96$ & $8.94 / 7.43$ & 63.59 & 17.29 \\
\hline & 34 & 1500 & 5107 & $9.49 / 8.11$ & $8.88 / 7.81$ & 72.51 & 20.50 \\
\hline & 35 & 1500 & 5730 & $10.95 / 9.74$ & $10.52 / 9.26$ & 67.49 & 17.81 \\
\hline \multicolumn{4}{|c|}{ Total (problems $28-35$ ) } & $62.57 / 54.16$ & $61.71 / 53.80$ & 356.32 & 115.82 \\
\hline \multirow{4}{*}{ 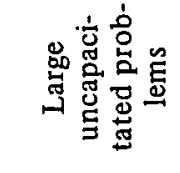 } & 37 & 5000 & 23000 & $87.05 / 73.64$ & $74.67 / 66.66$ & 681.94 & 281.87 \\
\hline & 38 & 3000 & 35000 & $68.25 / 57.84$ & $55.84 / 47.33$ & 607.89 & 274.46 \\
\hline & 39 & 5000 & 15000 & $89.83 / 75.17$ & $66.23 / 58.74$ & 558.60 & 151.00 \\
\hline & 40 & 3000 & 23000 & $50.42 / 42.73$ & $35.91 / 30.56$ & 369.40 & 174.74 \\
\hline \multicolumn{4}{|c|}{ Total (problems $37-40$ ) } & $295.55 / 249.38$ & $232.65 / 203.29$ & 2217.83 & 882.07 \\
\hline
\end{tabular}




\section{Table 2}

Large Assignment and Transportation Problems. Times in secs on VAX 11/750. All problems obtained using NETGEN, as described in the text. RELAX-II and RELAXT-II compiled under VMS 4.1; RNET compiled under VMS 3.7. Problems marked with * were obtained by NETGEN, and then, to make to problem feasible, an increment of 2 was added to the supply of each source node, and the demand of each sink node. Problems marked with ${ }^{+}$were similarly obtained, but the increment was 10

\begin{tabular}{|c|c|c|c|c|c|c|c|c|c|}
\hline No. & $\begin{array}{c}\text { Problem } \\
\text { type }\end{array}$ & $\begin{array}{l}\text { No. of } \\
\text { sources }\end{array}$ & $\begin{array}{l}\text { No. of } \\
\text { sinks }\end{array}$ & $\begin{array}{c}\text { No. of } \\
\text { arcs }\end{array}$ & $\begin{array}{l}\text { Cost } \\
\text { range }\end{array}$ & $\begin{array}{l}\text { Total } \\
\text { supply }\end{array}$ & RELAX-II & RELAXT-II & RNET \\
\hline 1 & \multirow{5}{*}{ 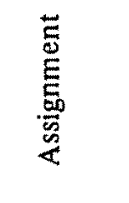 } & 1000 & 1000 & 8000 & $1-10$ & 1000 & 4.68 & 4.60 & 79.11 \\
\hline 2 & & 1500 & 1500 & 12000 & $1-10$ & 1500 & 7.23 & 7.03 & 199.44 \\
\hline 3 & & 2000 & 2000 & 16000 & $1-10$ & 2000 & 12.65 & 9.95 & 313.64 \\
\hline 4 & & 1000 & 1000 & 8000 & $1-1000$ & 1000 & 9.91 & 10.68 & 118.60 \\
\hline 5 & & 1500 & 1500 & 12000 & $1-1000$ & 1500 & 17.82 & 14.58 & 227.57 \\
\hline 6 & .5 & 1000 & 1000 & 8000 & $1-10$ & 100000 & 31.43 & 27.83 & 129.95 \\
\hline $7^{\star}$ & 焉 & 1500 & 1500 & 12000 & $1-10$ & 153000 & 60.86 & 56.20 & 300.79 \\
\hline $8^{+}$ & 5 & 2000 & 2000 & 16000 & $1-10$ & 220000 & 127.73 & 99.69 & 531.14 \\
\hline $9^{+}$ & 总 & 2500 & 2500 & 20000 & $1-10$ & 275000 & 144.66 & 115.65 & 790.57 \\
\hline $10^{+}$ & $\stackrel{5}{\rightleftarrows}$ & 3000 & 3000 & 24000 & $1-10$ & 330000 & 221.81 & 167.49 & 1246.45 \\
\hline 11 & 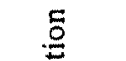 & 1000 & 1000 & 8000 & $1-1000$ & 100000 & 32.60 & 31.99 & 152.17 \\
\hline $12^{\star}$ & 苞 & 1500 & 1500 & 12000 & $1-1000$ & 153000 & 53.84 & 54.32 & 394.12 \\
\hline $13^{*}$ & 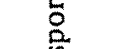 & 2000 & 2000 & 16000 & $1-1000$ & 220000 & 101.97 & 71.85 & 694.32 \\
\hline $14^{+}$ & 窎 & 2500 & 2500 & 20000 & $1-1000$ & 275000 & 107.93 & 96.71 & 1030.35 \\
\hline $15^{+}$ & 昰 & 3000 & 3000 & 24000 & $1-1000$ & 330000 & 133.85 & 102.93 & 1533.50 \\
\hline $16^{+}$ & .5 & 500 & 500 & 10000 & $1-100$ & 15000 & 16.44 & 11.43 & 84.04 \\
\hline $17^{*}$ & 㱏 & 750 & 750 & 15000 & $1-100$ & 22500 & 28.30 & 18.12 & 176.55 \\
\hline $18^{+}$ & 5 & 1000 & 1000 & 20000 & $1-100$ & 30000 & 51.01 & 31.31 & 306.97 \\
\hline $19^{+}$ & 品 & 1250 & 1250 & 25000 & $1-100$ & 37500 & 71.61 & 38.96 & 476.57 \\
\hline $20^{+}$ & $\stackrel{\Xi}{E}$ & 1500 & 1500 & 30000 & $1-100$ & 45000 & 68.09 & 41.03 & 727.38 \\
\hline
\end{tabular}



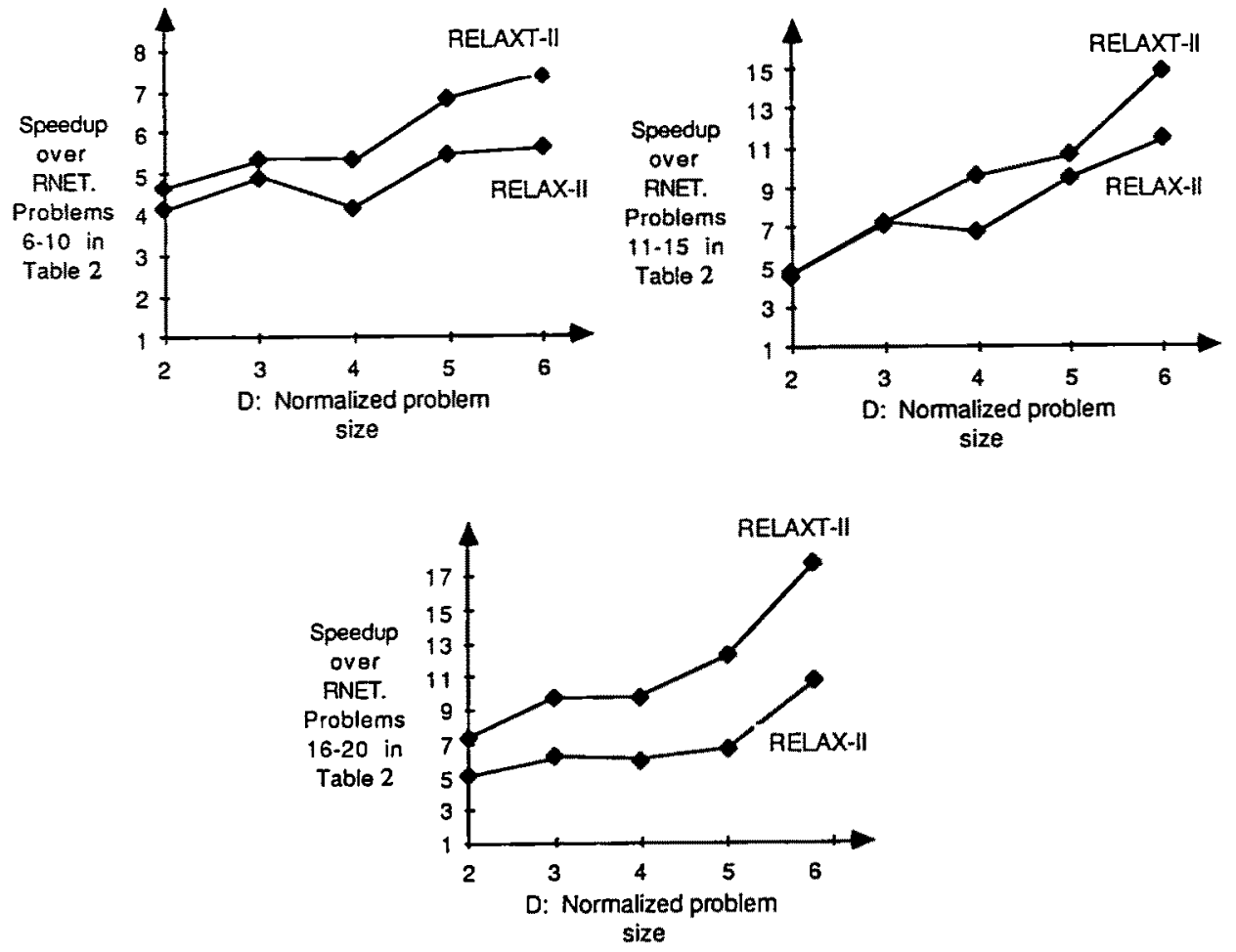

Fig. 5. Speedup factor of RELAX-II and RELAXT-II over RNET for the transportation problems of table 2 . The normalized dimension $D$ gives the number of nodes $\mathcal{N}$ and arcs $\&$ as follows:

$$
\begin{aligned}
& |\mathcal{N}|=1000 \star D,|\mathscr{A}|=4000 \star D \text { for problems } 6-15 \\
& |\mathcal{N}|=500 \star D,|\mathscr{A}|=5000 \star D \text { for problems } 16-20
\end{aligned}
$$

To corroborate the results of table 2, the random seed number of NETGEN was changed, and additional problems were solved using some of the problem data of the table. The results were qualitatively similar to those of table 2 . We also solved a set of transhipment problems of increasing size generated by our random problem generator called RANET. The comparison between RELAX-II, RELAXT-II and RNET is given in fig. 6. More experimentation and/or analysis is needed to establish conclusively the computational complexity implications of these experiments.

\section{Conclusions}

Relaxation methods adapt nonlinear programming ideas to solve linear network flow problems. They are much faster than classical methods on standard benchmark problems and a broad range of randomly generated problems. They are also better 


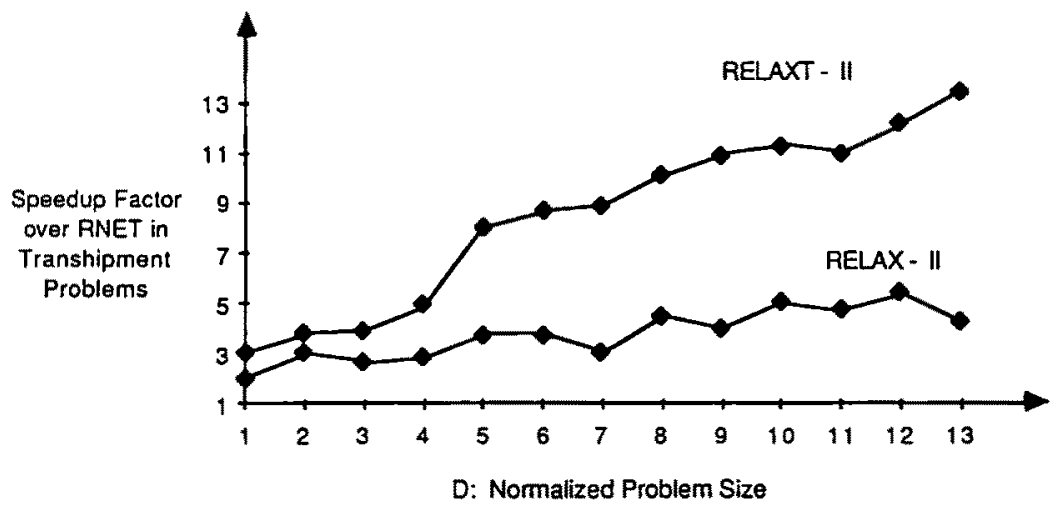

Fig. 6. Speedup factor of RELAX-II and RELAXT-II over RNET in lightly capacitated transhipment problems generated by our own random problem generator RANET. Each node is a transhipment node, and it is either a source or a sink. The normalized problem size $D$ gives the number of nodes and arcs as follows

$$
|\mathcal{N}|=200 \star D,|\otimes| \mid=3000 \star D \text {. }
$$

The node supplies and demands were drawn from the interval $[-1000,1000]$ according to a uniform distribution. The arc costs were drawn from the interval $[1,100]$ according to a uniform distribution. The arc capacities were drawn from the interval $[500,3000]$ according to a uniform distribution.

suited for post optimization analysis than primal-simplex. For example, suppose a problem is solved, and then is modified by changing a few arc capacities and/or node supplies. To solve the modified problem by the relaxation method, we use as starting node prices the prices obtained from the earlier solution, and we change the arc flows that violate the new capacity constraints to their new capacity bounds. Typically, this starting solution is close to optimal and solution of the modified problem is extremely fast. By contrast, to solve the modified problem using primal-simplex, one must provide a starting basis. The basis obtained from the earlier solution will typically not be a basis for the modified problem. As a result, a new starting basis has to be constructed, and there are no simple ways to choose this basis to be nearly optimal.

The main disadvantage of relaxation methods relative to primal-simplex is that they require more computer memory. However, technological trends are such that this disadvantage should become less significant in the future.

Our computational results provided some indication that relaxation has a superior average computational complexity over primal-simplex. Additional experimentation with large problems and/or analysis are needed to provide an answer to this important question.

The relaxation approach applies to a broad range of problems beyond the class considered in this paper (see [10-13]), including general linear programming problems. It also lends itself to distributed or parallel computation (see $[10,13-16]$ ). 
The relaxation codes RELAX-II and RELAXT-II together with other support programs, including a reoptimization and sensitivity analysis capacity, are in the public domain with no restrictions, and can be obtained from the authors at no cost on IBM-PC or Macintosh diskette.

\section{References}

[1] D.P. Bertsekas, A unified framework for minimum cost network flow problems, LIDS Report LIDS-P-1245-A, M.I.T. (1982); also Math. Progr. 32(1985)125.

[2] D.P. Bertsekas and P. Tseng, Relaxation methods for minimum cost - ordinary and generalized network flow problems, LIDS Report LIDS-P-1462, M.I.T. (1985); also Oper. Res. Journal, to appear.

[3] L.R. Ford, Jr. and D.R. Fulkerson, Flows in Networks (Princeton University Press, New Jersey, 1962).

[4] D.P. Bertsekas, A new algorithm for the assignment problem, Math. Progr. 21(1982)152.

[5] H.A. Aashtiani and T.L. Magnanti, Implementing primal-dual network flow algorithms, Oper. Res. Center Report 055-76, M.I.T. (1976).

[6] T. Magnanti, Optimization for sparse systems, in: Sparse Matrix Computations, ed. J.R. Bunch and D.J. Rose (Academic Press, New York, 1976) pp. 147-176.

[7] M.D. Grigoriadis and T. Hsu, The Rutgers minimum cost network flow subroutines (RNET documentation), Dept. of Computer Science, Rutgers University (1980).

[8] J. Kennington and R. Helgason, Algorithms for Network Programming (Wiley, New York, 1980).

[9] D. Klingman, A. Napier and J. Stutz, NETGEN - A program for generating large scale (un)capacitated assignment, transportation and minimum cost flow network problems Management Science 20(1974)814.

[10] D.P. Bertsekas, P. Hosein and P. Tseng, Relaxation methods for network flow problems with convex arc costs, SIAM J. Control and Optimization 25(1987).

[11] P. Tseng, Relaxation methods for monotropic programs, Ph.D. Thesis, M.I.T. (1986).

[12] P. Tseng and D.P. Bertsekas, Relaxation methods for linear programs, LIDS Report LIDSP-1553, M.I.T. (1986); also Math. of Oper. Res. 12(1987).

[13] P. Tseng and D.P. Bertsekas, Relaxation methods for problems with strictly convex separable costs and linear constraints, LIDS Report LIDS-P-1567, M.I.T. (1986); also Math. Progr. 38(1987).

[14] D.P. Bertsekas, Distributed relaxation methods for linear network flow problems, Proc. 25th IEEE Conf. on Decision and Control, Athens, Greece (1986).

[15] D.P. Bertsekas and D. El Baz, Distributed asynchronous relaxation methods for convex network flow problems, LIDS Report LIDS-P-1417, M.I.T. (1984); also SIAM J. Control and Optimization 25(1987)74.

[16] D.P. Bertsekas and J. Eckstein, Distributed asynchronous relaxation methods for linear network flow problems, Proc. of IFAC '87, Munich, Germany (1987) (Pergamon Press, Oxford). 


\section{THE BASIC ALGORITHM}

$l^{*}$ Read in problem data. " $/$

$n n:=$ number of nodes in network

na: = number of arcs in network

$l^{*}$ The nodes are numbered from 1 to $n n$ and the ares from 1 to na. $" /$

for arc : $=1$ to na do

$$
\begin{aligned}
& \operatorname{cost}(\operatorname{arc}):=\text { cost of arc } \\
& \text { upbd(arc) }:=\text { flow upper bound of arc } \\
& \text { head(arc) }:=\text { head node of arc } \\
& \text { tail(arc) }:=\text { tail node of arc }
\end{aligned}
$$

end do

for node $:=1$ to $\mathrm{nn}$ do

dfet(node) : = extraneous flow supply out of node

end do

$/$ Initialize dual prices to 0 and then assign flow to arcs to satisfy complementary slackness. $/$ for arc: $=1$ to na do

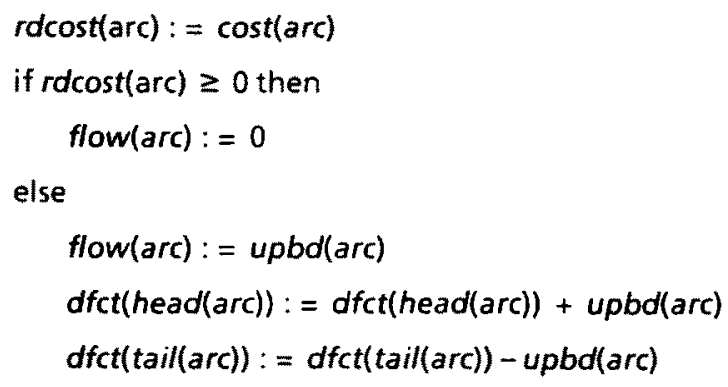

end do

I" Start relaxation iterations. "/

while $\operatorname{dfct}(i) \neq 0$ for some $i$ do

for node $:=1$ to $n$ n do

$$
\begin{aligned}
& \text { if } \operatorname{dfct}(\text { node })>0 \text { then } \\
& \text { pred(node) }:=0 \\
& \text { labelset }:=\{\text { node }\} \\
& \text { scanset }:=\{\varnothing\} \\
& \text { augnode }:=0 \\
& \text { ascent }:=\text { false }
\end{aligned}
$$


while augnode $=0$ and not ascent do

Choose a node $1 \in$ labelset $\backslash$ scanset

scanset $:=$ scanset $\cup\{$ node 1$\}$

$I^{*}$ Start scanning step. *I

scanning(node 1 ,augnode)

$I^{*}$ Check if scanset corresponds to a dual ascent direction. "I

if

$\sum \operatorname{dfct}\left(\right.$ node1) $>\quad \sum$ flow(arc) $+\sum$ upbd(arc)-flow(arc)

node $1 \in$ scanset $r d \operatorname{cost}(\operatorname{arc})=0$ head(arc) $€$ scanset rdcost $(a r c)=0$

tail(arc) scanset head(arc) $R$ scanset tail(arc) $\in$ scanset

then ascent: = true

end do

if ascent then

doascent

else

augflow(augnode, node)

end do

end do

procedure scanning(node1, augnode)

$l^{*}$ This procedure performs a scanning step at node $1 . *$

for all arc such that head $(a r c)=$ node 1 do

if $r$ cost $(a r c)=0$ and flow $(a r c)>0$ then

node $2:=$ tail $($ arc $)$

if node2 labelset then

pred(node2) : = arc

labelset : = labelset $\mathrm{U}$ \{node2\}

if $\mathrm{dfct}($ node 2$)<0$ then augnode : = node 2

end do

for all arc such that tail(arc) $=$ node $1 \mathrm{do}$

if $r d \cos t(a r c)=0$ and flow $(a r c)<u p b d(a r c)$ then 


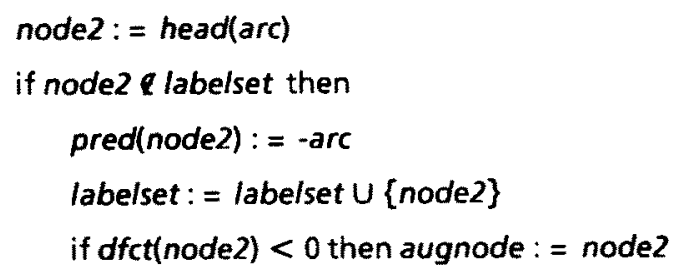

end do

end;

procedure doascent

$l^{*}$ This procedure performs dual ascent by line-minimization and updates the flow accordingly to satisfy complementary slackness. $*$

while

$\sum \operatorname{dfct}\left(\right.$ node1) $>\sum$ flow(arc) $+\sum$ upbd(arc)-flow(arc)

node $1 \in$ scanset

$$
r d \operatorname{cost}(a r c)=0
$$

$r d \cos t(a r c)=0$

head(arc) $\epsilon$ scanset

head(arc) e scanset

tail(arc) $\&$ scanset

tail(arc) $\in$ scanset

do

/" Compute the stepsize to the next breakpoint in the dual cost and decrease the price of all nodes in scanset by the stepsize. Adjust the arc flow accordingly to maintain complementary slackness. "/

pricechange : = very large positive number

for all arc such that head(arc) $€$ scanset and tail(arc) scanset do

if $r d \cos t(a r c)=0$ then

$$
\begin{aligned}
& d f c t(\text { head(arc) }):=d f c t(\text { head }(a r c))-\text { flow(arc) } \\
& d f c t(t a i l(a r c)):=d f c t(t a i l(a r c))+\text { flow(arc) } \\
& \text { flow(arc) : }=0
\end{aligned}
$$

if $0<-r d \operatorname{cost}(a r c)<$ pricechange then pricechange : = -rdcost(arc)

end do

for all arc such that head(arc) 1 scanset and taill(arc) $€$ scanset do

if $r$ cost $(a r c)=0$ then

$$
\begin{aligned}
& d f c t(\text { head }(\operatorname{arc})):=d f c t(\text { head }(\operatorname{arc}))+(\text { upbd(arc) }- \text { flow }(\operatorname{arc})) \\
& \operatorname{dfct}(\operatorname{tail}(\operatorname{arc})):=d f c t(\text { tail }(\operatorname{arc}))-(\text { upbd(arc)-flow }(\operatorname{arc})) \\
& \text { flow(arc) }:=\text { upbd(arc) }
\end{aligned}
$$

if $0<r d c o s t(a r c)<$ pricechange then pricechange : = rdcost(arc)

end do

for all arc such that head(arc) $€$ scanset and taillarc) $\ell$ scanset do 


$$
\text { rdcost(arc) : = rdcost }(a r c)+\text { pricechange }
$$

end do

for all arc such that head(arc) $f$ scanset and tail(arc) $€$ scanset do

$$
\text { rdcost }(\operatorname{arc}):=\operatorname{rdcost}(\operatorname{arc})-\text { pricechange }
$$

end do

end do

end;

procedure augflow(augnode, node)

$I^{\star}$ This procedure adjusts the flow on arcs to decrease the total deficit, while maintaining complement slackness. *I

flowchange $:=\min \{\operatorname{dfct}($ node), $-\operatorname{dfct}($ augnode) $\}$

node1 : = augnode

while node $1 \neq$ node do

arc $:=\operatorname{pred}($ node 1$)$

if are $>0$ then

flowchange $:=\min \{$ flowchange, flow $(a r c)\}$

node $1:=$ head $($ arc $)$

else

flowchange $:=\min \{$ flowchange, upbd(-arc)-flow(-arc) $\}$

node $1:=$ tail(-arc)

end do

$\operatorname{dfct}$ (node) : = dfct(node) - flowchange

dfct(augnode) $:=\operatorname{dfct}($ augnode $)+$ flowchange

node $1:$ = augnode

while node 1 \& node do

arc $:=\operatorname{pred}($ node 1$)$

if arc $>0$ then

$$
\begin{aligned}
& \text { flow }(a r c):=\text { flow }(a r c)-\text { flowchange } \\
& \text { node1 }:=\text { head(arc) }
\end{aligned}
$$

else

$$
\begin{aligned}
& \text { flow }(-a r c):=\text { flow }(-a r c)+\text { flowchange } \\
& \text { node } 1:=\text { tail(-arc) }
\end{aligned}
$$

end do

end: 



\section{Appendix}

\begin{tabular}{|c|c|c|}
\hline C & $* * * * * * *$ & SAMFLE CALLING FFIOGFAM FOF SUBFIOUTINE FELAXT ******** \\
\hline C & $* * *$ & (MINIMUM COST NETWOFK: FLOW FFOELEM) \\
\hline C & $* * *$ & **** \\
\hline C & $* * *$ & THE FFIOGFIAM IS BASED ON THE FAFER \\
\hline C & $* * *$ & D.F. EEFTSEKAS, P.TSENG "THE FELAX CODES FOR \\
\hline C & $* * *$ & LINEAR MINIMUM COST NETWOFK FLOW FROELEMS" \\
\hline C & *** & ANNALS OF OPERATIONS RESEARCH, THIS VOLUME \\
\hline C & $* * *$ & 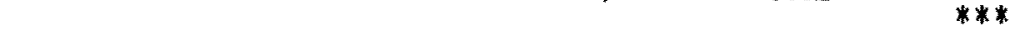 \\
\hline C & $* * *$ & ALL THE BUEROUTINES AFE WRITTEN IN STANDARD \\
\hline C & $* * *$ & FOFTEIANT7. \\
\hline $\mathrm{c}$ & $* * *$ & $* * *$ \\
\hline C & $* * *$ & QUESTIONS AND COMMENTS SHOULD EE DIFECTED TO \\
\hline $\mathcal{E}$ & *** & DIMITFI EEFTSEKAS AND FAUL TSENG \\
\hline $\mathrm{C}$ & *** & DEF'ARTMENT OF ELECTFIICAL ENGINEEFING \& \\
\hline C & $* * *$ & COMFUTEF SCIENCE \\
\hline C. & $* * *$ & LAEOFATOFYY FOF: INFOFIMATION AND DECISION SYSTEMS \\
\hline C & $* * *$ & M. I.T. CAMEFIIDGE, MASSACHUSETTS, 02139, U.S.A. \\
\hline & ***** & 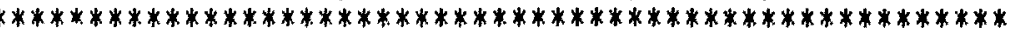 \\
\hline
\end{tabular}

THIS PROGFAM WILL READ AND SOLVE A FROELEM FILE CFEATED VIA THE RANDOM FROELEM GENERATOR NETGEN OR ANY GENERATOR THAT USES THE NETGEN FORMAT.

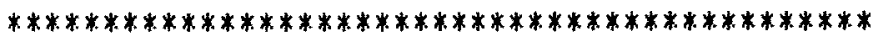

DOUELE FFECISION TCOST

INTEGER C (70000), X(70000), U (70000), F:C (70000), E (6000)

INTEGER CAF (70000), STARTN (70000), ENDN (70000)

INTEGEF I $1(6000)$, I $2(6000)$, IS $(6000)$, I $4(70000)$, I $5(6000)$

INTEGER I6 (70000), I $7(70000)$

LOGICAL L1 (6000), L 2(6000), TEST, FEFEEAT

COMMON /ARFIAYS/STARTN/ARFAYE/ENDN/ARFAYU/U/ARRAYX/X

*/ARRAY9/FC/ARRAYE/E/ELK1/I1/ELK2/I2/ELK3/IS/BLK4/I4/ELK5/15/BLK6

*/I6/BLKT/I7/ELKB/L1/BLK9/L2/L/N,NA, LARGE

COMMON/ELKR/REFEAT/ARRAYC/C/BLKCAF/CAP

COMMON /ELK10/TFSTOU/ELK11/TNXTOU/ELK12/TFSTIN/ELK13/TNXTIN

C

DIMENSION TFSTOU (6000), TNXTOU(70000), TFSTIN(6000), TNXTIN(70000)

FEAD (5,*)N, IA

FEAD $(5, *)$ (STARTN $(I), I=1, I A)$

FEAD $(5, *)$ (ENDN (I), I=1,IA)

READ $(5, *)(C(I), I=1, I A)$

FEAD $(5, *)(U(I), I=1, I A)$

DO $10 \quad \mathrm{I}=1, \mathrm{~N}$

$10 B(I)=0$

$M=0$

DO $20 \quad I=1$, IA

IF (STARTN(I) .EQ.N+1) THEN

$E(E N D N(I))=-U(I)$

ELSE IF (ENDN(I).EQ.N+2) THEN $B(S T A R T N(I))=U(I)$

ELSE

$M=M+1$

$C(M)=C(I)$ 


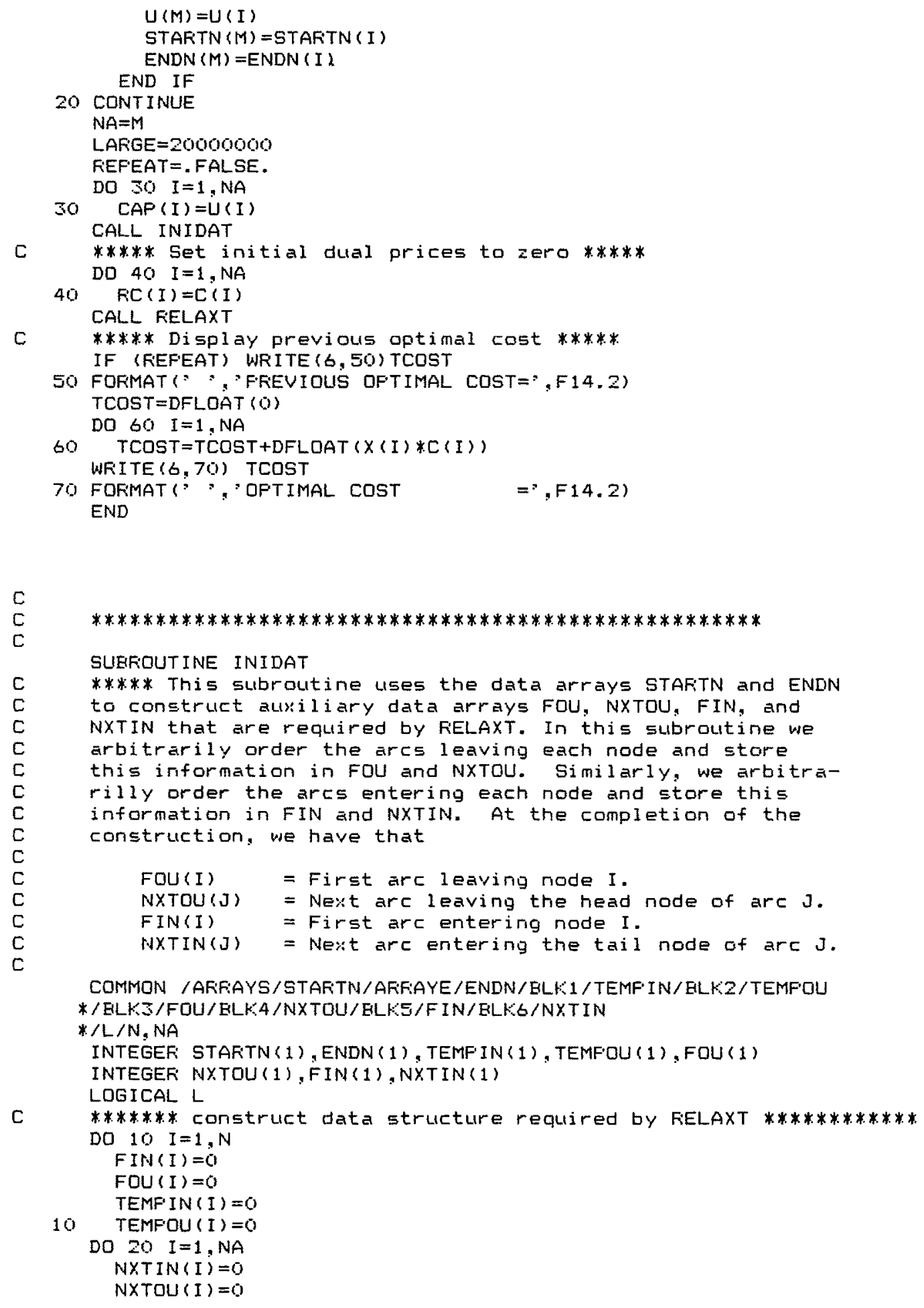




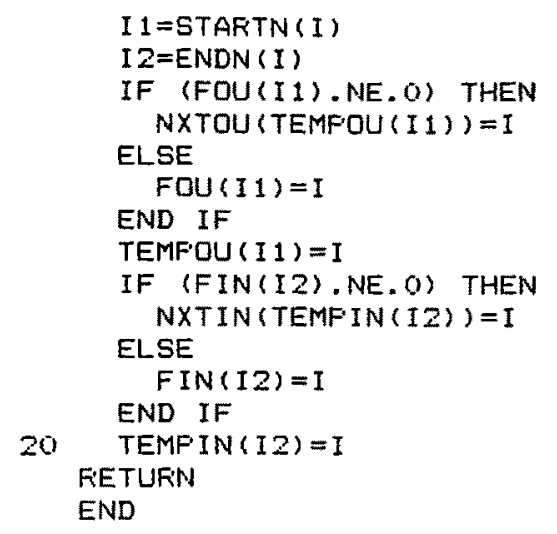

SUEFIOUT INE FELAXT

FELEASE AFR. 1988

This subroutine solves the minimum (Iinear) cost ordinary network flow problem.

The routine implements the relaxation method of

Bertsekas, D. F., "A Unified Framework for Firimal-Dual Methods ..." Math. Frogramming, Vol. 32, 1985, pp. 125-145

Eertsekas, D. F., \& Tseng, F. "Kel axation Methods for Minimum ..." Operations Fesearch J., 1987 (to appear)

Bertsekas, D. F., \& Tseng, F., "The FELAX Codes for Linear Minimum Cost Network Flow Froblems", ANNALS OF OPERATIONS RESEARCH, THIS VOLUME

The routine was written by Dimitri Eertsekas and Faul Tseng with contributions by Jonathan Eckstein.

This code is in the public domain and can be used for any purpose. It can be distributed freely.

Users are requested to acknowledge the authorship

of the code, and the relaxation algorithm. No modifications should be made to this code other than the minimal necessary to make it compatible with the FORTRAN compilers of specific machines. When reporting computational results please be sure to describe the memory limitations of your machine. Generally FELAXT requires more memory than primal simplex codes and may penalized severely by limited machine memory.

The difference between this routine and the similar code fiELAX is that it maintains a data structure that gives all the balanced arcs in the network. This structure is called the "tree" for historical reasons, Even though it describes a subnetwork that will generally be neither acyclic nor connected. Also, the tree may contain some arcs that are not bal anced: it turns out to be cheaper to purge arcs that have become unbalanced only when their end nodes are being scanned, as opposed to always maintaining an exact set of balanced arcs. 


\section{******************************************************************}

The user must supply the following inputs to the subroutine:

All data should be in INTEGEF*4. To run in limited memory systems the arrays STARTN, ENDN, NXTIN, NXTOU, SAVE, FIN, FOU, LABEL, FRDCSF may be decl ared as INTEGEF*2.

$N$ (the number of nodes)

NA (the number of arcs)

LARGE (a very large positive integer to represent infinity. All problem data should be less than LAFGE in magnitude, and LAFGE should be less than, say, $1 / 4$ the largest INTEGER*4 of the machine used. This wili guard primarily against overflow in uncapacitated problems where the arc capacities are taken finite but very large.)

STARTN(NA) (the head node array)

ENDN(NA) (the tail node array)

FC (NA) (the reduced cost array)

$X$ (NA) (the arc flow array)

$U(N A)$ (the arc flow capacity array)

DFCT (N) (the deficit array)

FOU (N) (the first arc out array)

FIN(N) (the first are in array)

NXTOU (NA) (the next are out array)

NXTIN(NA) (the next arc in array)

This subroutine places the optimal flow in the array $x$

and the corresponding reduced cost vector in the array $R C$.

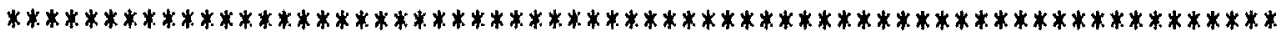

IMFLICIT INTEGER (A-Z)

LOGICAL REFEAT, FEASEL, QUIT, SCAN, SWITCH, MAFK, FOSIT, FCHANG

COMMON/ARFRAYS/STARTN/ARRAYE/ENDN/AFRAYU/U/AFRAYX/X/ARRRAYQ/FiC

*/ARRAYE/DFCT/ELK1/LAEEL/ELKZ/FFDCSF/ELK3/FOU/ELK4/NXTOU/ELK5/FIN

*/BLKG/NXTIN/ELK7/SAVE/BLK8/SCAN/ELK9/MAFK/L/N, NA, LARGE

*/ELKFI/REFEAT

COMMON /BLK1O/TFSTOU/ELK11/TNXTOU/ELK12/TFSTIN/ELK13/TNXTIN

Each common block contains just one array, so the arrays in RELAXT can be dimerisioned to 1 element and take their dimension from the main calling routine. With this trick FELAXT need not be recompiled if the problem dimension changes. If your FORTKAN does not support this feature change the dimensions below to be the same as the ones declared in your main calling program.

DIMENSION TFSTOU (1), TNXTOU(1), TFSTIN(1), TNXTIN(1)

DIMENSION STARTN(1), ENDN (1), U(1), $X(1), F C(1), \operatorname{DFCT}(1)$

DIMENSION LAEEL (1), FFDCSF( 1 ), SCAN (1), FOU (1), NXTOU (1)

DIMENSION FIN(1), NXTIN(1), SAVE (1), MAFK (1)

$C$
$C$
$C$
$C$
$C$
$C$
$C$
$C$

DDFOS and DDNEG are arrays that give the directional derivatives for all positive and negative single-node price changes. These are used only in the initial phase of the algorithm, before the "tree" datastructure comes into play. Therefore, they are equivalenced to TFSTOU and TFSTIN, which are the same size (number of nodes) and are only used after the tree comes into use. 


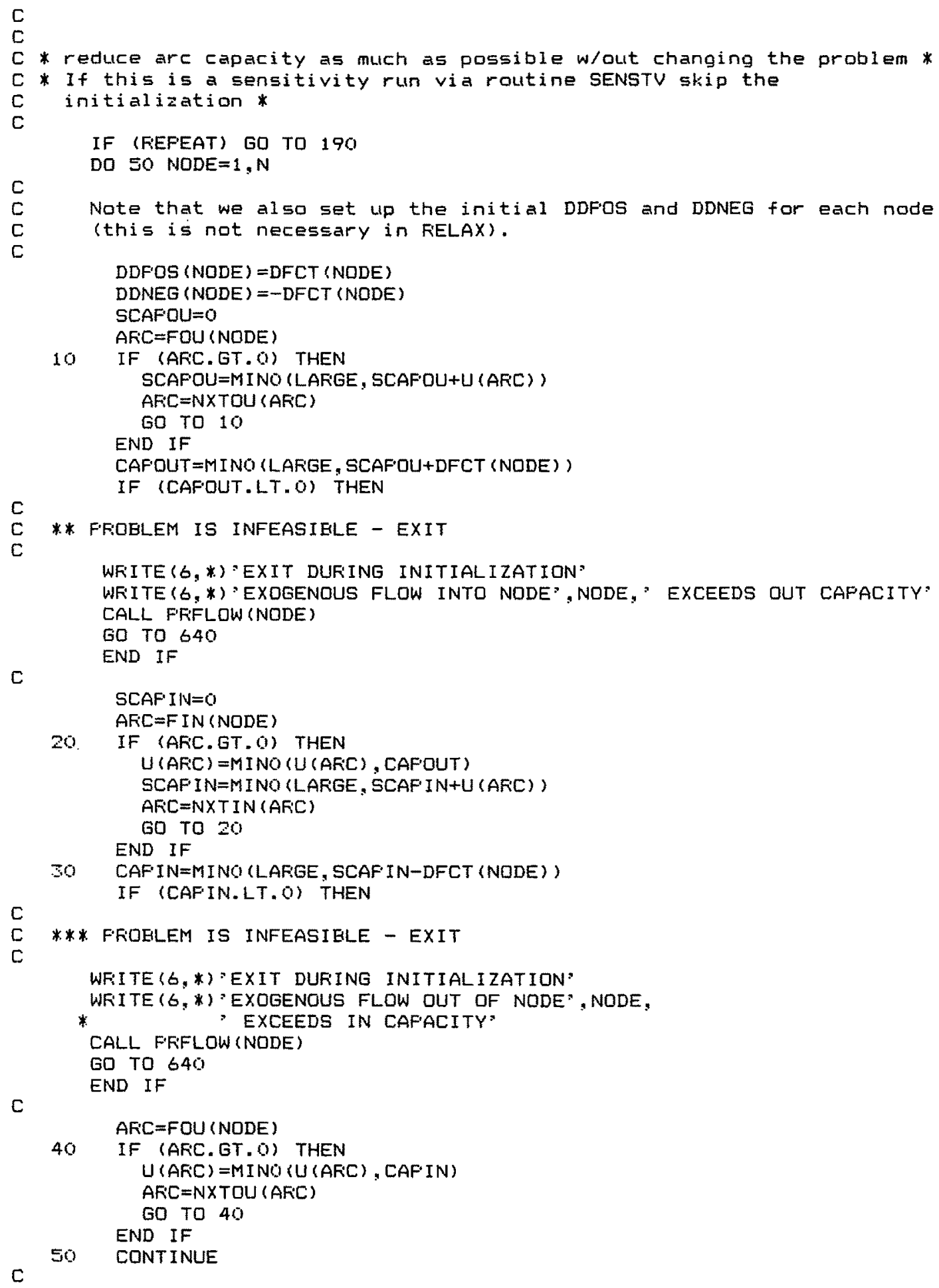




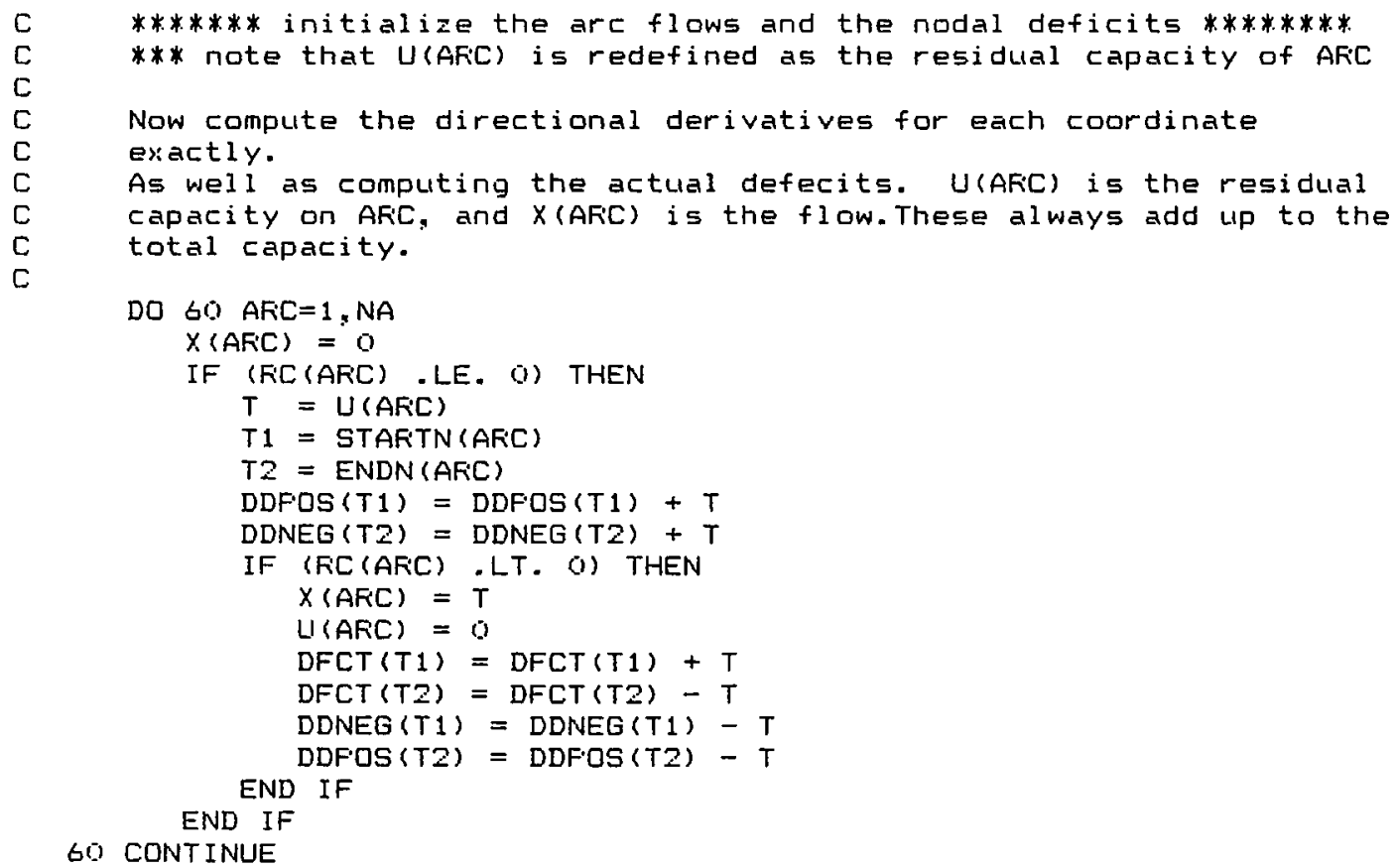

C
c
c
c
c

Adaptive strategy: the number of strictly single-node iteration passes attempted is a function of the average density of the network.

IF (NA.GT.N*10) THEN NF'ASS $=2$

ELSE NFASS $=3$

END IF

We now do 2 or 3 passes through all the nodes. This is the initial phase:if a single node iteration is not possible, we just go on to the next node.

DO 180 FASSES $=1$, NFASS

DO 170 NODE $=1, \mathrm{~N}$

IF (DFCT (NODE) . NE. O) THEN

Frice rise or price drop? (Note: it is impossible to have both.)

IF (DDFOS(NODE) . LE. (1) THEN

Frice rise. Loop over breakpoints in tFrice(NODE) direction.

On outgoing arcs, tension will rise and reduced cost will fall - so, next break comes at smallest positive reduced cost.

On incoming arcs, tension will fall and reduced cost will rise -- so, neyt break, comes at smallest negative reduced cost.

$$
\text { DELFFIC = LAFIGE }
$$

$A F C=F Q U(N O D E)$

IF (AFIC . GT. O) THEN

TFiC $=$ FiC (AFiC)

IF ( (TFE . GT. O) . AND. (TKC . LT. DELFRC) ) THEN 


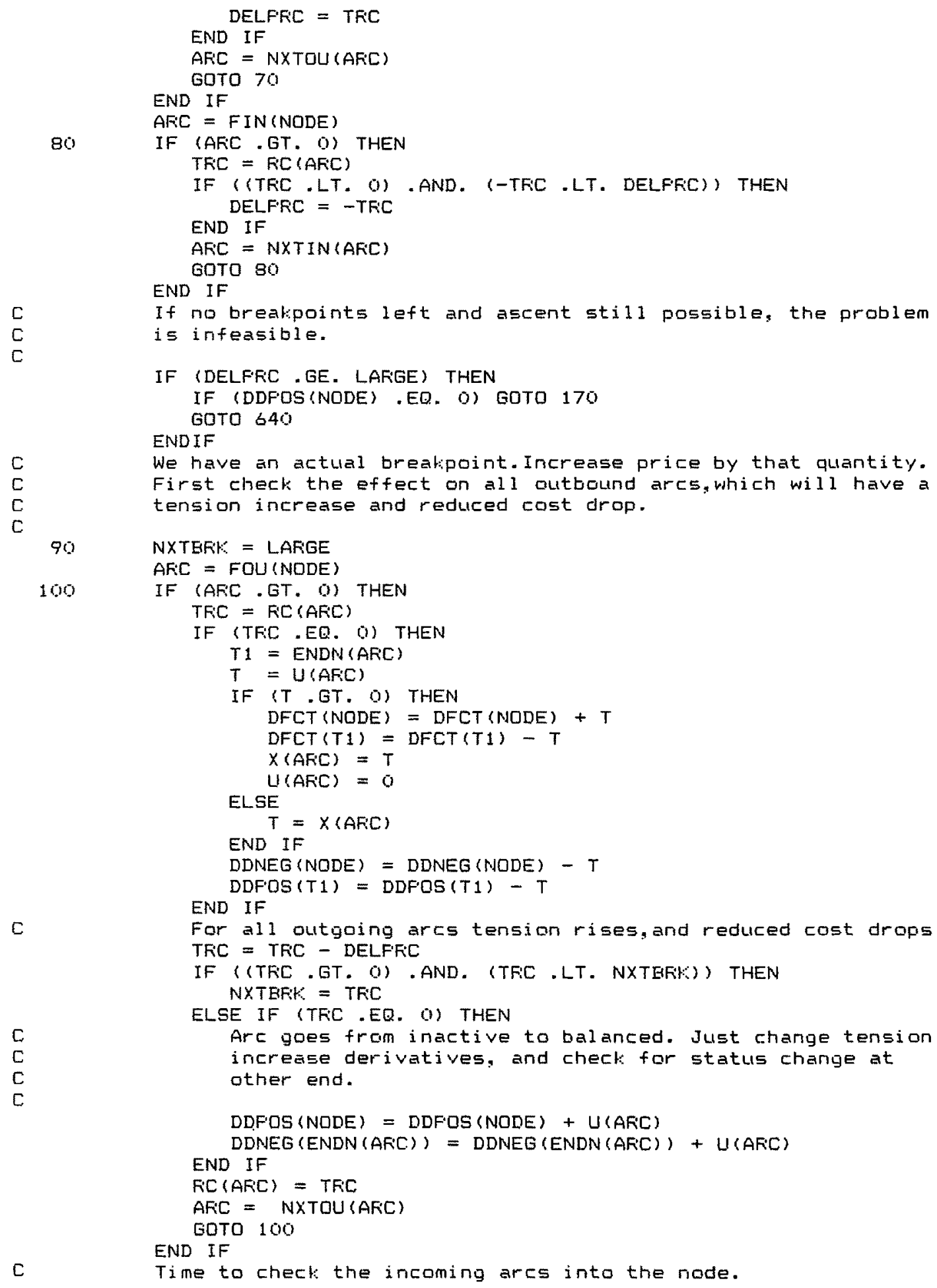

Time to chect: the incoming arcs into the node. 
$C$
$C$

110

These arcs will have an tension decrease and a reduced cost rise.

$A R C=F I N(N D D E)$

IF (ARC. . GT: O) THEN

$T F C=F I C(A F C)$

IF (TRC .EQ. O) THEN

$T 1=\operatorname{STARTN}(A R C)$

$T=X(A F C)$

IF (T . GT. O) THEN

DFCT (NODE) = DFCT (NDDE) + T

$\operatorname{DFCT}(T 1)=\operatorname{DFCT}(T 1)-T$

$U(A F C)=T$

ELSE $X(A F C)=0$

END IF

$\operatorname{DDFOS}(T 1)=\operatorname{DDFOS}(T 1)-T$

END IF

DDNEG (NODE) = DDNEG (NODE) - T

C

Note the reduced cost $r$ ise for every arc.

$T R C=T R C+D E L F R C$

IF ( (TFC . LT. O) . AND. (-TRC . LT. NXTERK)) THEN NXTERK $=-T R C$

C

ELSE IF (TFC . EQ. (0) THEN

Now check for movement from active to balanced.

C

If so, tension decrease derivatives increase. DDNEG (STARTN $(A K C))=\operatorname{DDNEG}(S T A R T N(A R C))+X(A R C)$ DDFOS (NODE) $=$ DDFOS (NDDE) $+X$ (ARC)

END IF

FC $(A F C)=T F C$

$A F: C=N X T I N(A F C)$

GOTO 110

C

END IF

C

We are now done with the iteration. If the current direction

is still a (degenerate) descent direction, push onward.

IF ((DDFOS (NODE) .LE. 0) . AND. (NXTEFK . LT. LAFGE)) THEN DELFFC = NXTEFK

END IF GOTO 90

C Now comes the code for a price decrease at NODE.

C On outgoing arcs, tension will drop and reduced cost will increase

C

C

C - so, next break comes at smallest negative reduced cost.

On incoming arcs, tension will increase and reduced cost will fall

-- so, next break comes at smallest positive reduced cost.

C

ELSE IF (DDNEG (NODE) . LE. ()) THEN

DELFFC = LAFGE

$A F C=F Q U(N D D E)$

120 IF (AFC . GT. O) THEN

$T K C=R C$ (ARC)

IF ((TRC . LT. O) .AND. (-TRC . LT. DELFFE) ) THEN

ENDIF DELFRC $=-T R C$

$A F C=N \times T O U(A F C)$

ENDIF

GDTO 120

$A R C=F I N(N D D E)$

130

IF (AF:C . GT. O) THEN 


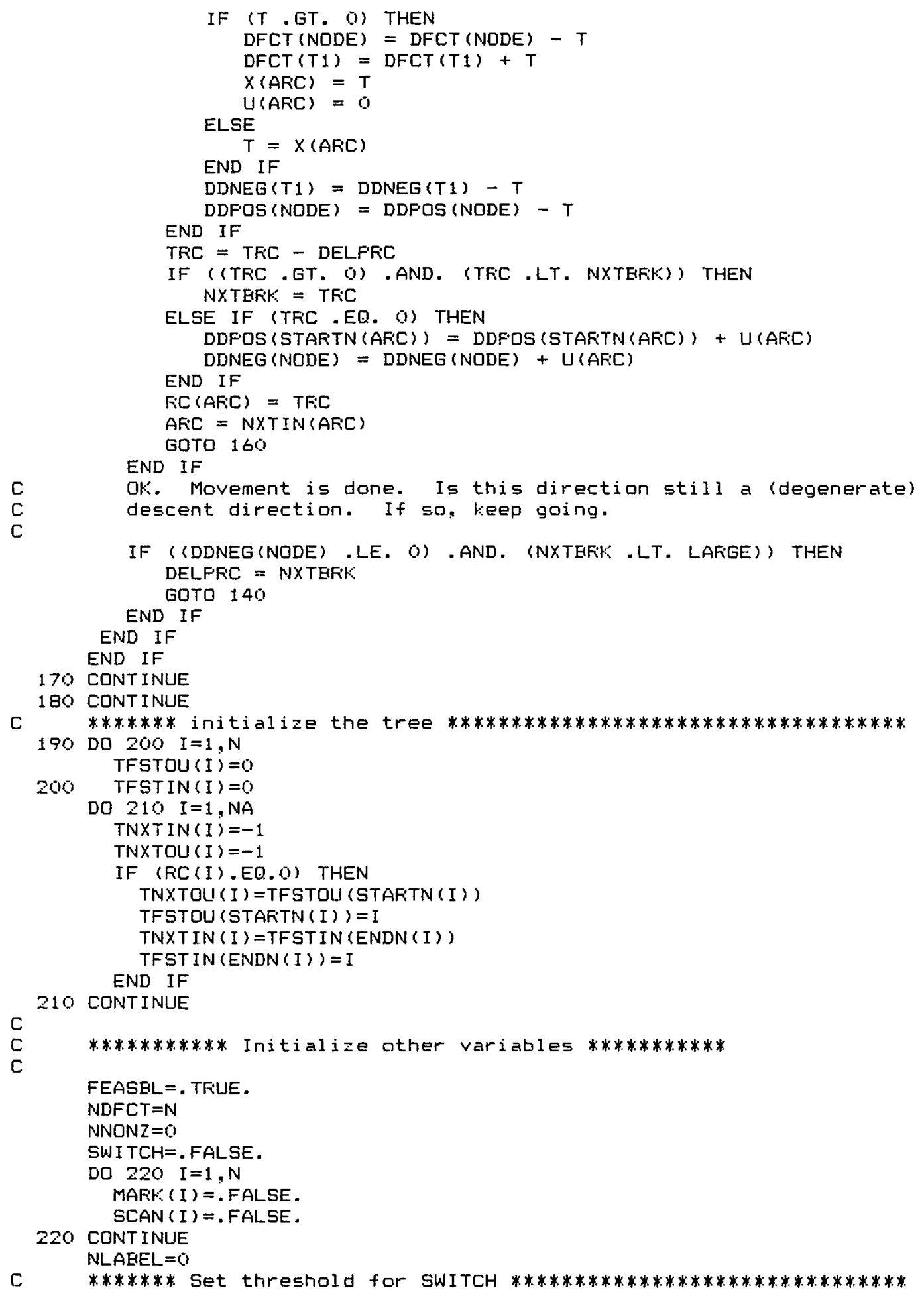




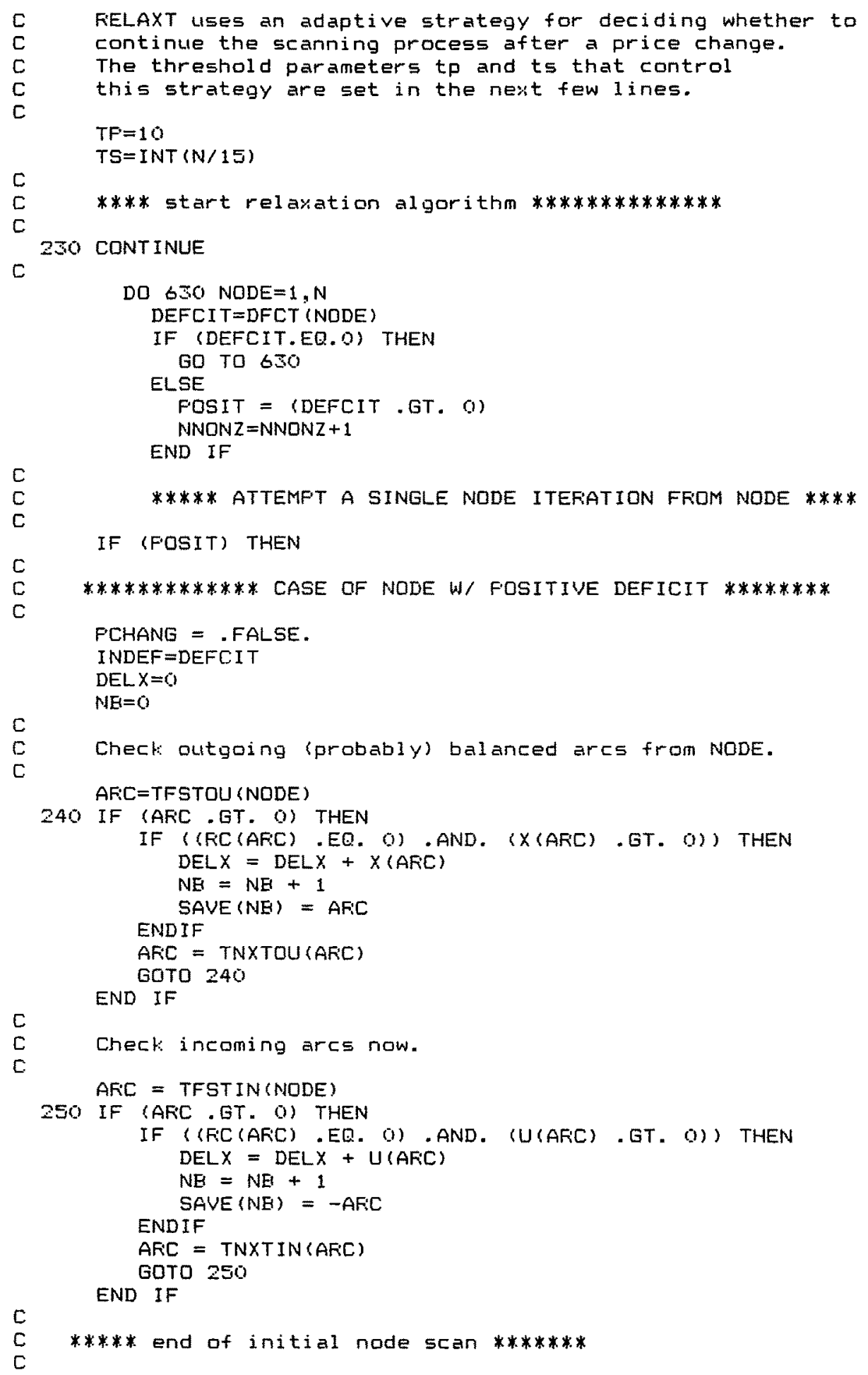




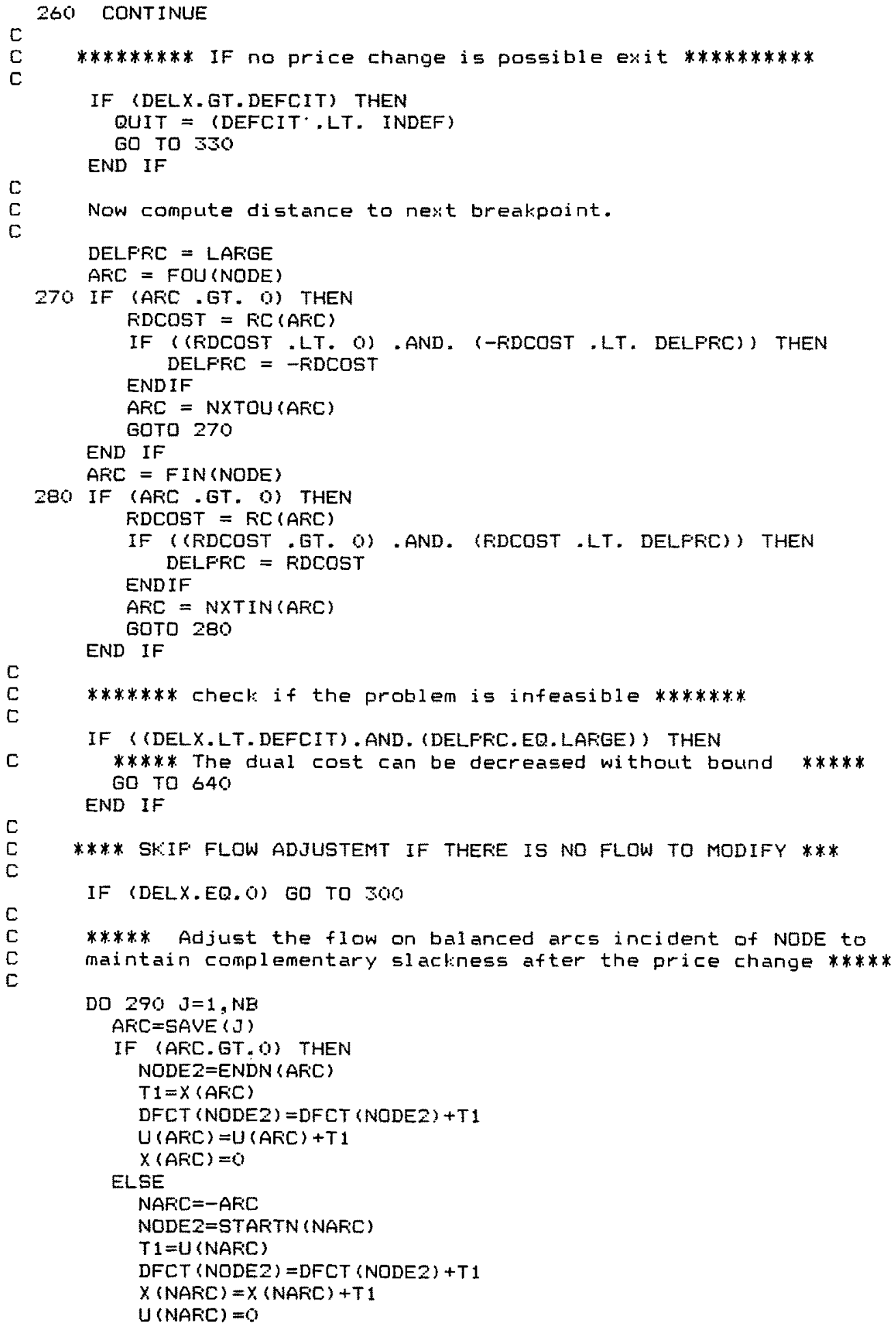




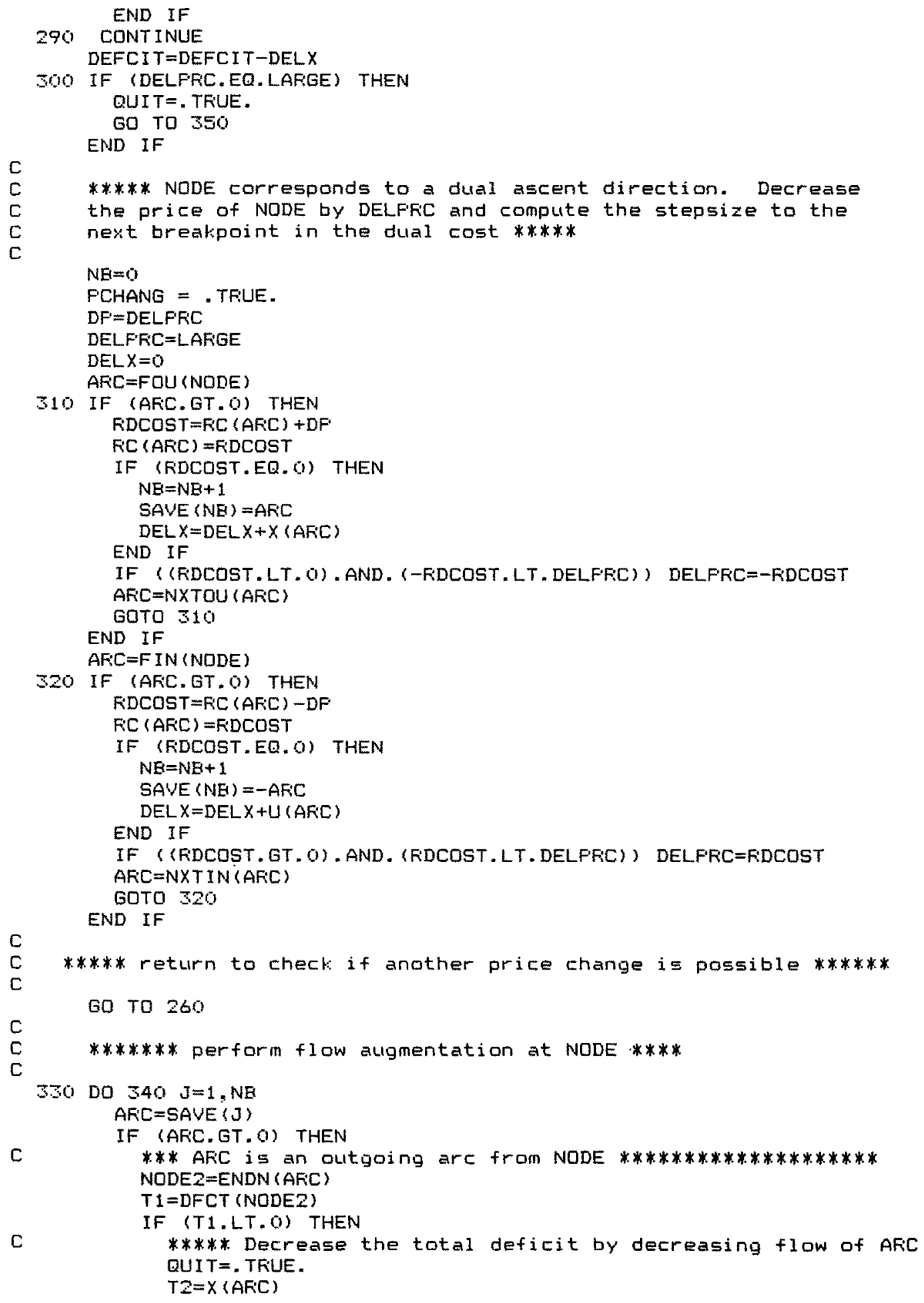


C

\section{ELSE}

$D X=M I N O(D E F C I T,-T 1, T 2)$

DEFCI T $=$ DEFCI $T-D X$

DFCT $($ NODE 2$)=T 1+D X$

$X(A F C)=T 2-D X$

$U(A F C)=U(A F C)+D X$

IF (DEFCIT.EQ.0) GO TO 350

END IF

*** -AFC is an incoming arc to NODE **********************

NARC $=-A R C$

NODE $2=5 T$ TARTN (NAFIC)

$T 1=$ DFCT $(N O D E Z)$

IF (T1.LT.O) THEN

C

***** Decrease the total deficit by increasing flow of -ARC

QUIT $=$, TFUE.

$T Z=U$ (NAFC)

$D X=M I N O$ (DEFCI $T,-T 1, T 2)$

DEFCI.T=DEFCIT-DX

DFCT $(N O D E 2)=T 1+D X$

$X(N A F C)=X($ NAFIC $)+D X$

$U(N A F C)=T 2-D X$

IF (DEFCIT.EQ.0) GO TO 350

END I
END IF
CONT INUE

C

340 CONTINUE

350 DFCT (NODE) $=$ DEFCI $T$

$\mathrm{C}$

Feconstruct the list of balanced arcs adjacent to this node.

First, the list at this node is now totally different. Eat the old lists of incoming and outgoing balanced arcs, and create

a whole new one. This way we get the in and out lists of balanced arcs for NODE to be exactly correct. For the adjacent nodes, we add in all the newly balanced arcs, but do not bother getting rid

$\mathrm{C}$ of formerly balanced ones (they will be purged the next time the

C

C

$\mathrm{C}$ adjacent node is s(anned).

IF (PCHANG) THEN

ARC = TFSTOU (NODE)

TFSTOU (NODE) $=0$

360 IF (ARC . GT. O) THEN

NXTAFC = TNXTOU $(A F C)$

TNXTOU (ARC) $=-1$

AF:C $=$ NXTARC

END IF

GOTO 360

ARC = TFSTIN (NODE)

370 TFSTIN(NODE) $=0$

IF (ARC . GT. O) THEN

NXTARC $=$ TNXTIN $(A R C)$

$\operatorname{TNXTIN}(A F C)=-1$

ARIC $=$ NXTARC

END IF

GOTO 370

$\mathrm{C}$

*** Now add the currently balanced arcs to the list for this

c

*** node (which is now empty), and the appropriate adjacent ones

DO $380 \mathrm{~J}=1, \mathrm{NE}$

$A R C=\operatorname{SAVE}(\mathrm{J})$

IF (AFC.LE.O) AF:C=-AFC 


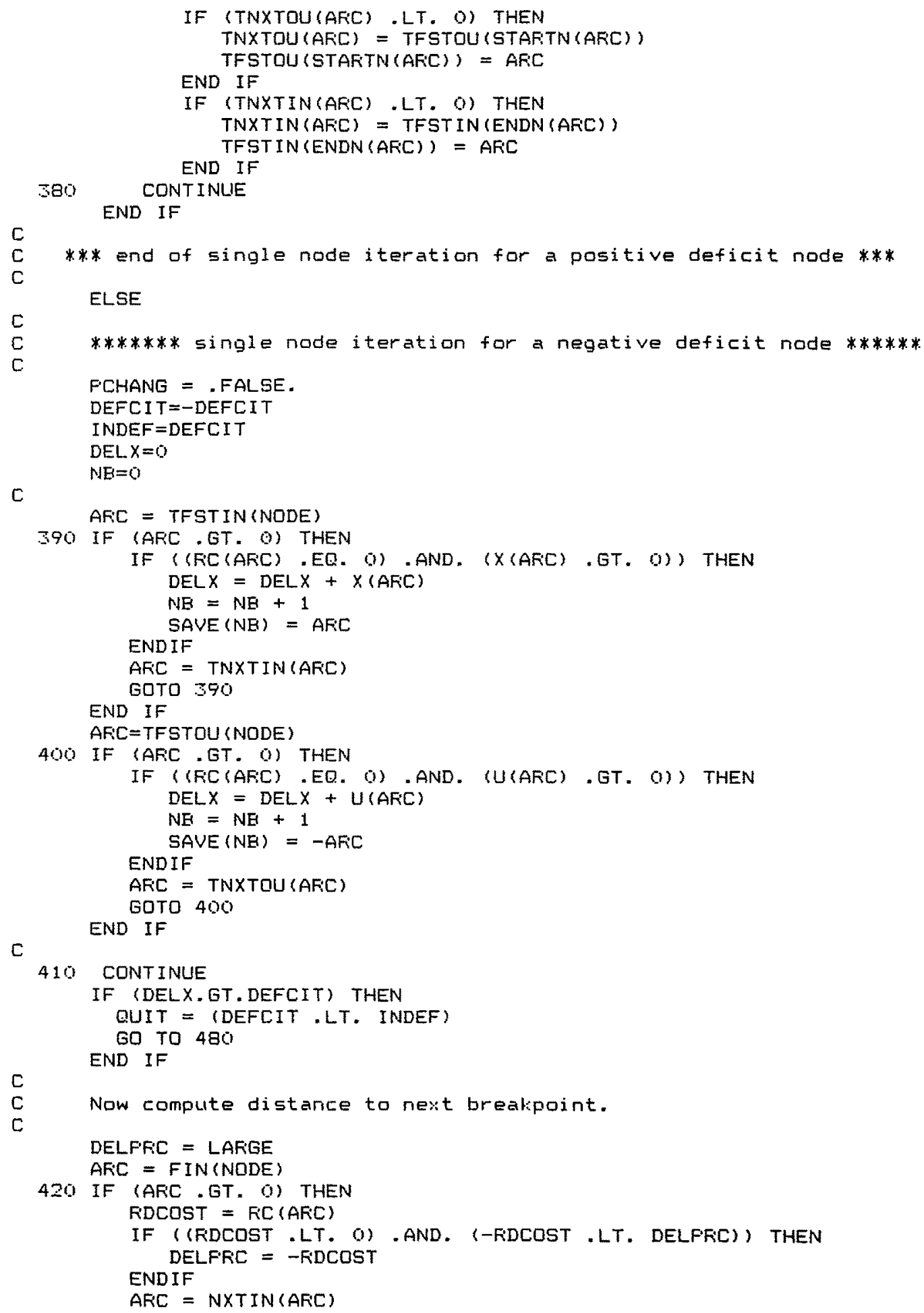




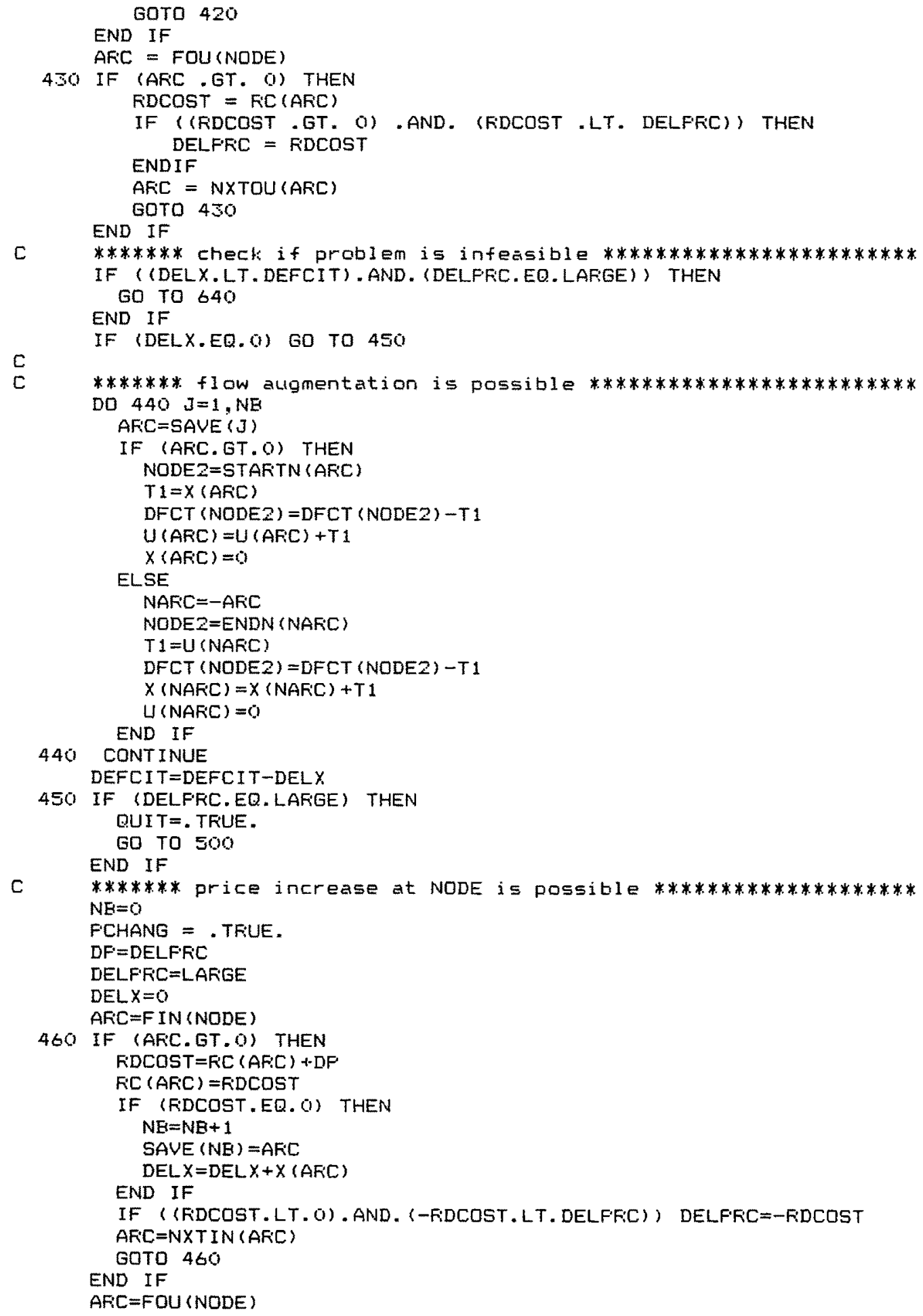




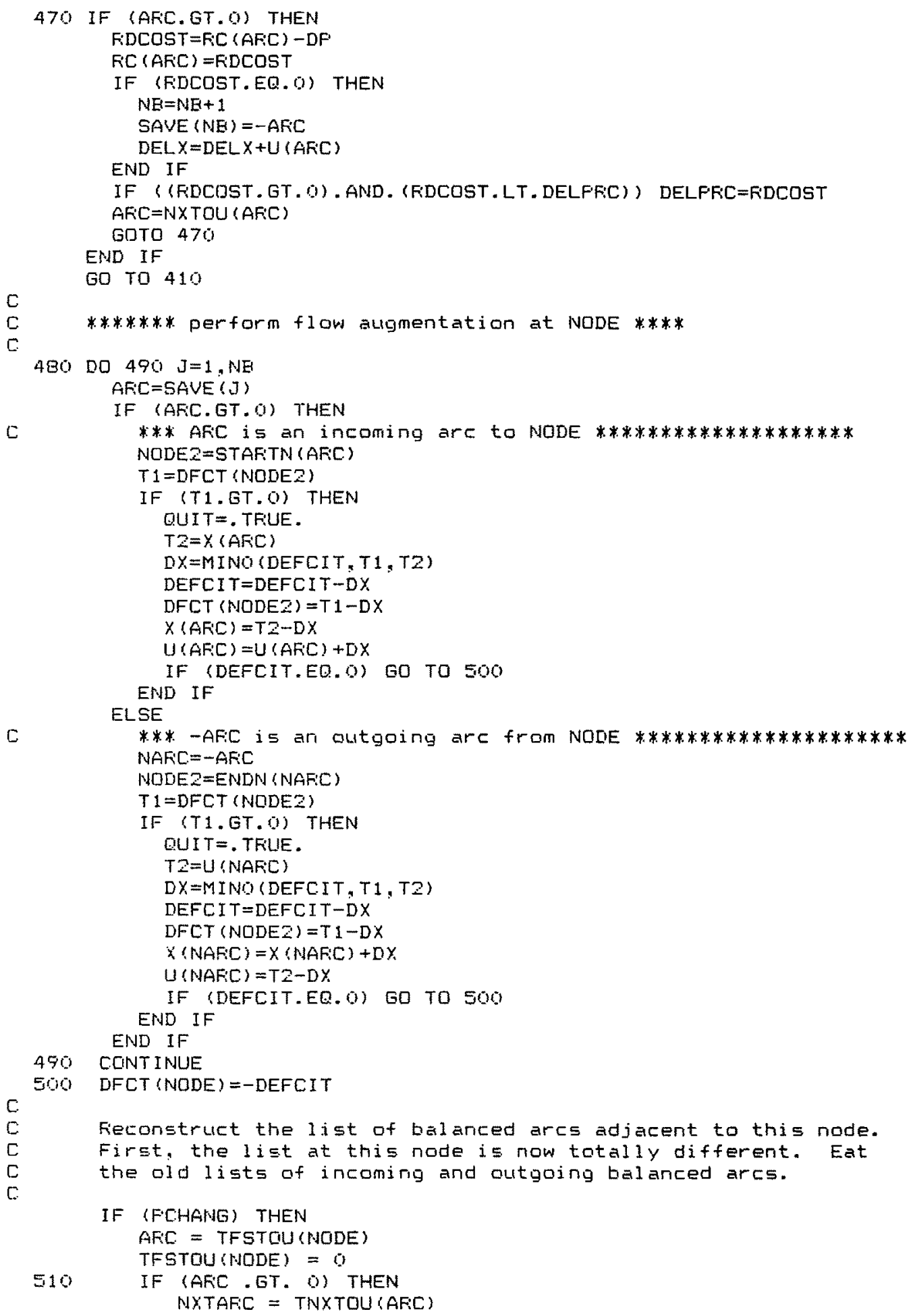




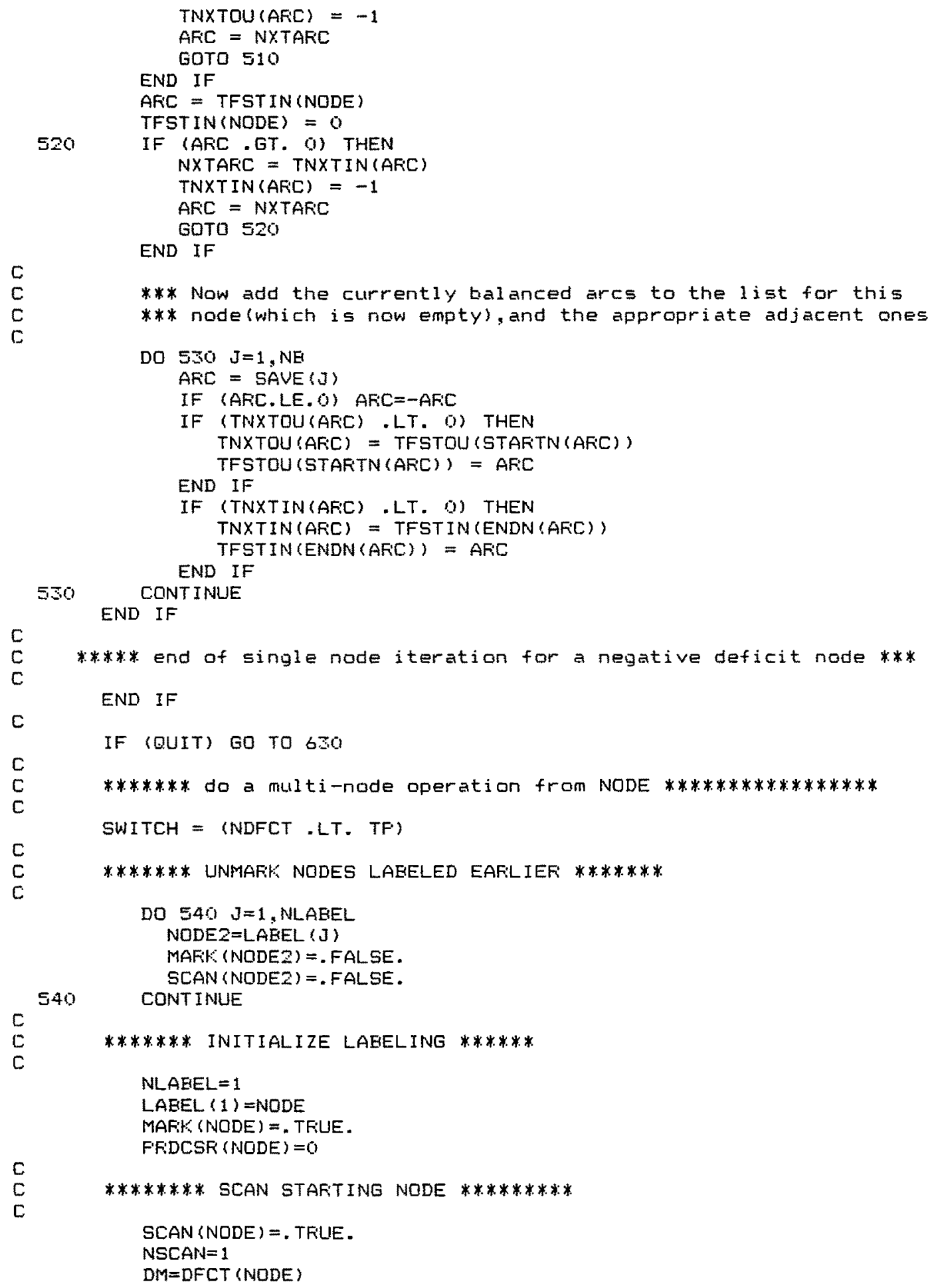




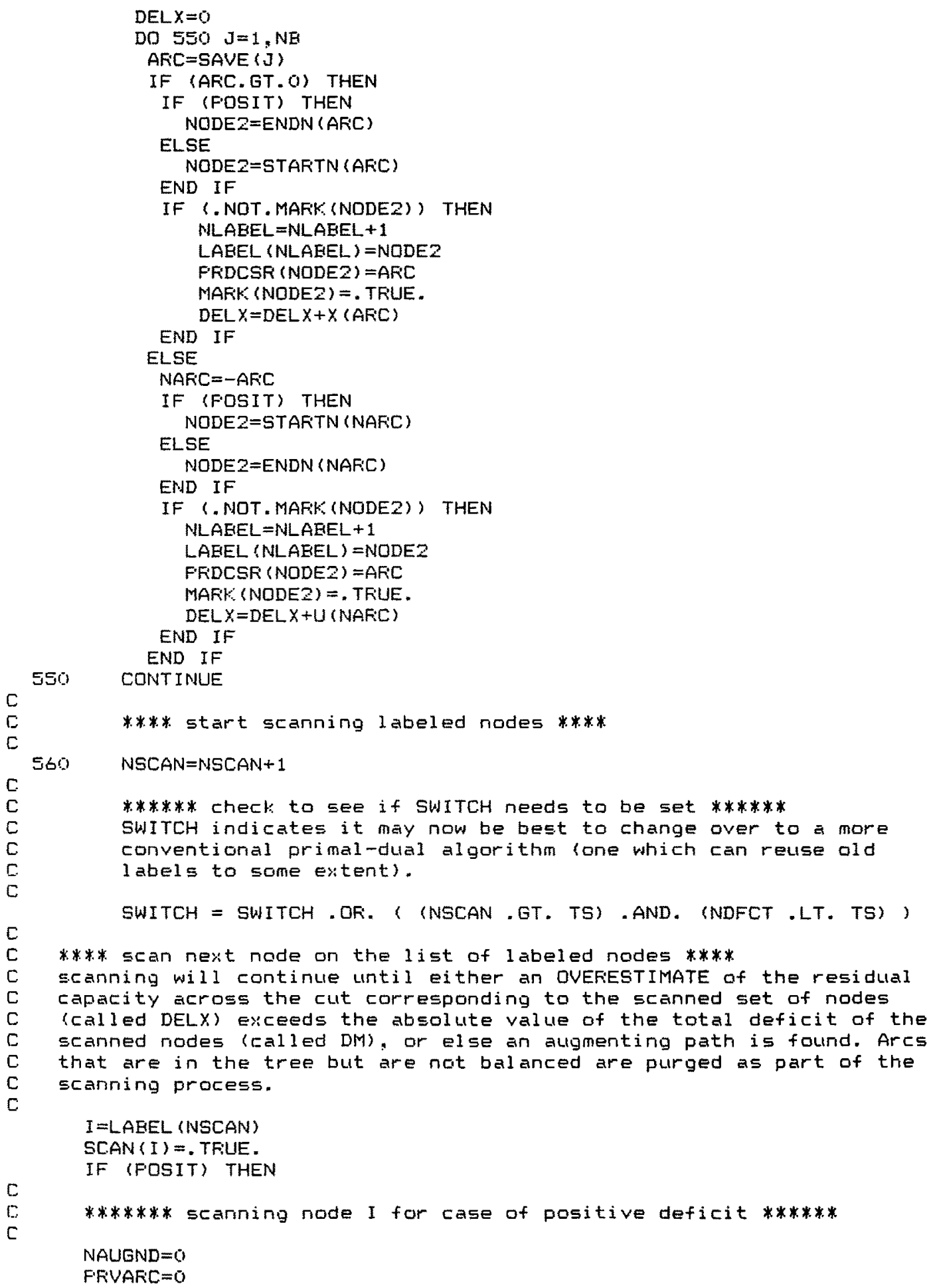


C

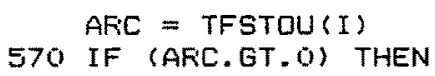

C

C

C

C

C

C

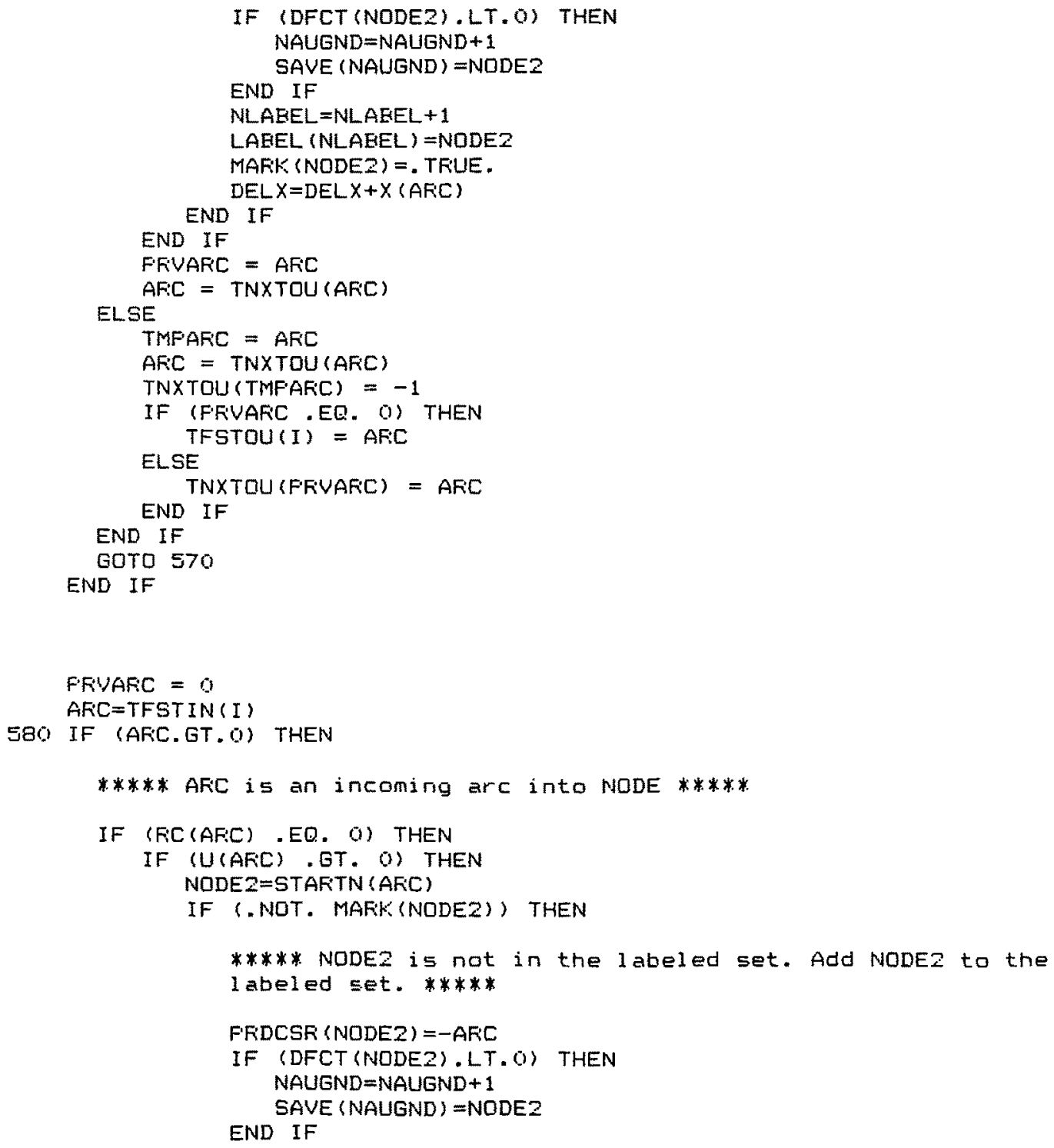

c 


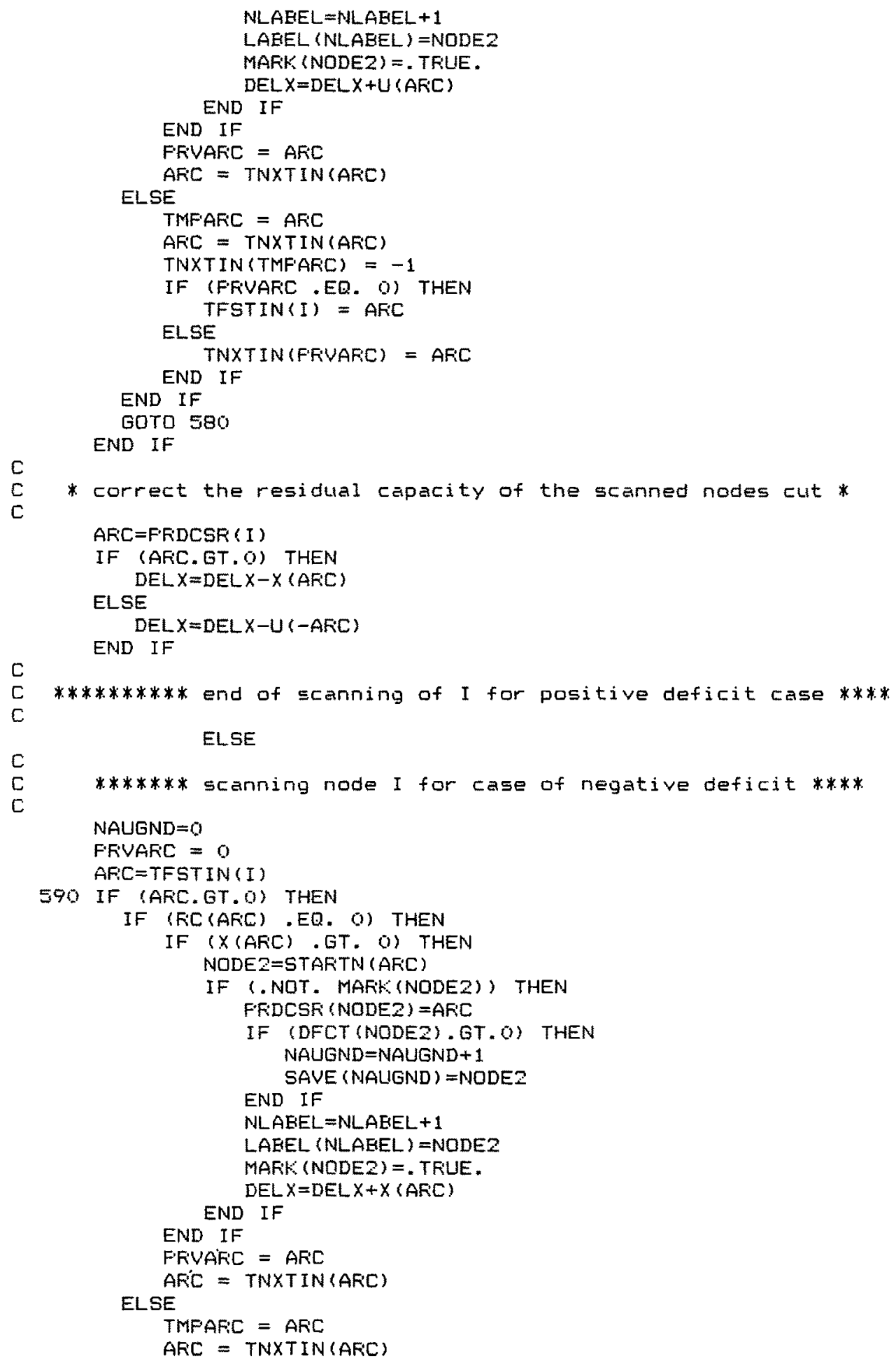




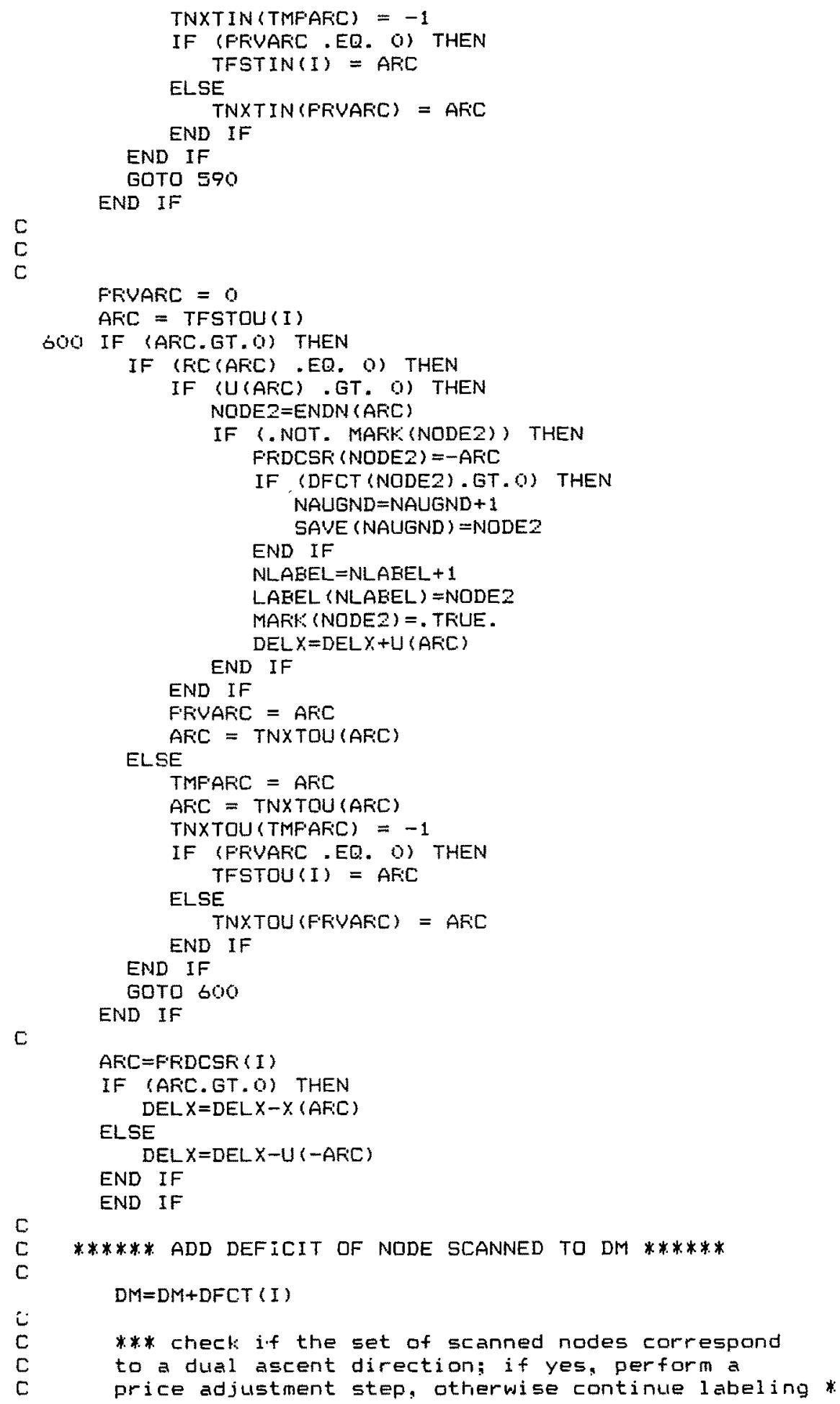


c

C

C

c

C

C

C

C

C

C

$\mathrm{C}$
$\mathrm{C}$
$\mathrm{C}$

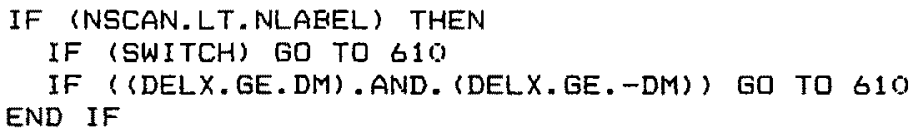




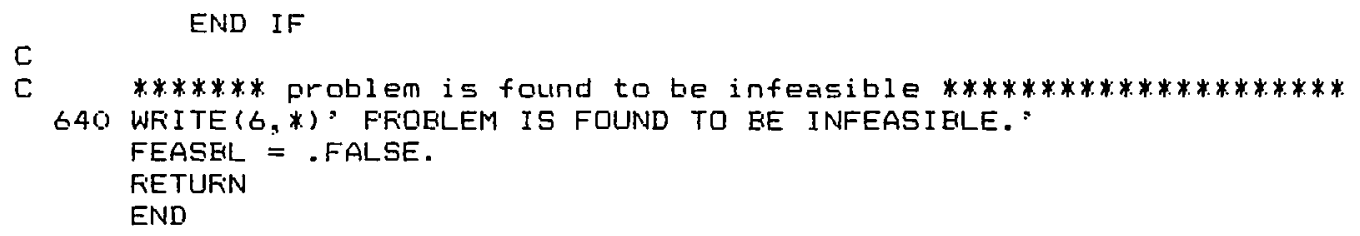

\section{SUEROUTINE FFFFLOW (NODE)}

C
C
C
c
C
C
C
c
c
c
C

***** This subroutine prints the deficit and the flows of arcs incident to NODE. It is used for diagnostic purpases in case of an infeasible problem here. It can be used also for more general diagnostic purposes. ******

IMFLICIT INTEGEF: $(A-Z)$

COMMON/AF:FAYS /STAFTN/AFRIAYE/ENDN/AFRAAYU/U/AFRAAYX/X

*. /AFFAYYE/DFCT/ELKS/FOU/ELK4/NXTOU/BLKE/FIN/ELKO/NXTIN

C

DIMENSION STAFTN (1), ENDN (1),U(1), $x(1)$, DFCT (1)

DIMENSION FQU (1), NXTOU(1)

DIMENSION FIN(1), NXTIN(1)

C

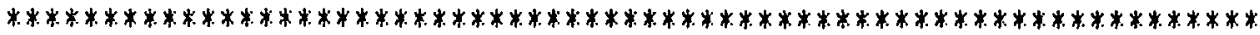

WFITE $(\sigma, *)$ ) DEFICIT (I.E., NET FLOW OUT) OF NODE $=*$, DFCT (NODE) WFITE $(6, *)$ 'FLOWS AND CAFACITIES OF INCIDENT AFICS OF NODE' NODE IF (FOU (NODE). EQ. O) THEN WFITE $(6, *)$ "NO OUTGLING AF:CS"

ELSE AF:C $=$ FOU $(N O D E)$

10 IF (AF:C. GT.O) THEN WFITE $(6, *)$ "AFIC" AF:C, " BETWEEN NDDES", NODE, ENDN (AFC) WFI ITE $(6, *)=F L O W=;, X(A F C)$ WFITE $(6, *)$ " RESIDUAL CAFACITY $=", U(A F C)$ AFIC $=$ N $\times$, TOU $(A F C)$ GO TO 10 END IF END IF

c

$\mathrm{C}$$$
*
$$

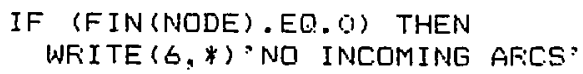


C

FEETUFN

END

SUEROUTINE AUGFL1 (AUGNOD)

$\mathrm{C}$

***** This subroutine performs the flow augmentation step.

A flow augmenting path has been identified in the scanning step and here the flow of all arcs positively (negatively) oriented in the flow augmenting path is decreased (increased) to decrease the total deficit. ******

IMFLICIT INTEGEF (A-Z)

COMMON/ARFAYS/STARTN/ARFAYE/ENDN/ARFAYU/U/AFRAYX/X

* / AFFIAYE/DFCT / ELKZ/FRDCSR

DIMENSION STARTN (1), ENDN(1),U(1), X(1), DFCT (1), FRDCSR (1)

$c$
$C$
$C$
$C$

***** A flow augmenting path ending at AUGNOD is found. Determine $D X$, the amount of flow change. *****

$D X=-D F C T$ (AUGNDD)

$I \mathrm{E}=$ AUIGNOD

10 IF (FFDCSF (IE). NE. O) THEN

$A F C=F F D C S F(I E)$

IF (AFC. GT. O) THEN

$D X=M I N O(D X, X(A R C))$

IE=STAFTN (ARC)

ELSE

$D X=M I N O(D X, U(-A F C))$

I $B=E N D N(-A F i C)$

END IF

GOTO 10

END IF

FOOT $=I B$

$D X=M I N O(D X, D F C T$ (ROOT) )

IF (DX .LE. O) RETUFN

$c$

******. Update the flow by decreasing (increasing) the flow of all arcs positively (negatively) oriented in the flow augmenting path. Adjust the deficits accordingly. *******

DFCT (AUGNOD) $=$ DFCT $($ AUGNOD $)+D X$

DFCT $($ FOOT $)=$ DFCT $($ ROOT $)-D X$

$I B=A U G N O D$

20 IF (IB.NE.ROOT) THEN

ARC $=$ FFDCSF $($ IE)

IF (AFC.GT. O) THEN

$X(A F: C)=X(A F C)-D X$

$U(A F: C)=U(A F C)+D X$

$I E=S T A F T N(A F I C)$

ELSE

NAF:C $=-A F C$

$X$ (NAFC $)=X$ (NAFC $)+D X$

$U(N A F C)=U($ NARC $)-D X$

I $E=E N D N$ (NAFC)

END IF

GOTO 20

END IF 
FETUFN

END

SUEROUTINE ASCNT1 (DM, DELX, NLABEL, AUGNOD, FEASEL, SWITCH, WNSCAN)

This subroutine essentially performs the multi-node price adjustment step. It first checlss if the set of scanned nodes correspond to a dual ascent direction. If yes, then decrease the price of all scanned nodes. There are two possibilities for price adjustment: If SWITCH=. TFiUE. then the set of scanned nodes corresponds to an elementary direction of maximal rate of ascent, in which case the price of all scanned rodes are decreased until the next breakpoint in the dual cost is encountered. At this point some arc becomes balanced and more node(s) are added to the labeled set.

If SWITCH=.FALSE. then the prices of all scanned nodes are decreased until the rate of ascent becomes negative ithis corresponds to the price adjustment step in which both the line search and the degenerate ascent iteration are implemented).

\section{IMFLICIT INTEGEF: $(A-Z)$}

The two "tree"-based ascent routines have a common temporary storage area whose dimension is set below. The maximum conceivable amount needed equals the number of arcs, but this should never actually occur.

LQGICAL SCAN, MAFE, SWITCH, FEASEL, QUIT

COMMON/AFEFAYS/STAFTN/AFFAYE/ENDN/AFFAYU/U/AFIFAYX/X/AFFIAYG /FE * /AFFAYE/DFCT/ELF1/LABEL/ELK2/FFDCSF/ELKIS/FOU/ELKK4/ *NXTOU/ELK5/F IN/BLK6/NXTIN/ELK7/SAVE/BLK8/SCAN/BLFG/MAFK:

*. L $/ N, N A, L A F E E$

COMNON/ELK1O/TFSTOU/BLK11/TNXTOU/ELK12/TFSTIN/ELK1S/TNXTIN COMMON /ASCELK/B

DIMENSION TFSTOU(1), TNXTOU(1), TFSTIN(1), TNXTIN(1)

DIMENSION STAFTN (1), ENDN (1), U(1), X(1), RC (1), DFCT (1), LAEEL (1)

DIMENSION FFDCSF(1), FOU(1), NXTOU(1), FIN(1), NXTIN(1)

DIMENSION SAVE (1), SCAN (1), MAFK (1)

****** store the arcs between the set of scanned nodes and its complement in SAVE and compute DELFRC, the stepsize to the next breakpoint in the dual cost in the direction of decreasing prices of the scanned nodes. *********

DELFFEC=LAFGE

$D L X=0$

NSAVE $=0$

$\mathrm{C}$

C

$\mathrm{C}$

C

*** calculate the array SAVE of arcs across the cut of scanned nodes in a different way depending on whether NSCANYN/2 or not. This is done for efficiency. *****

IF (NSCAN.LE.N/Z) THEN 


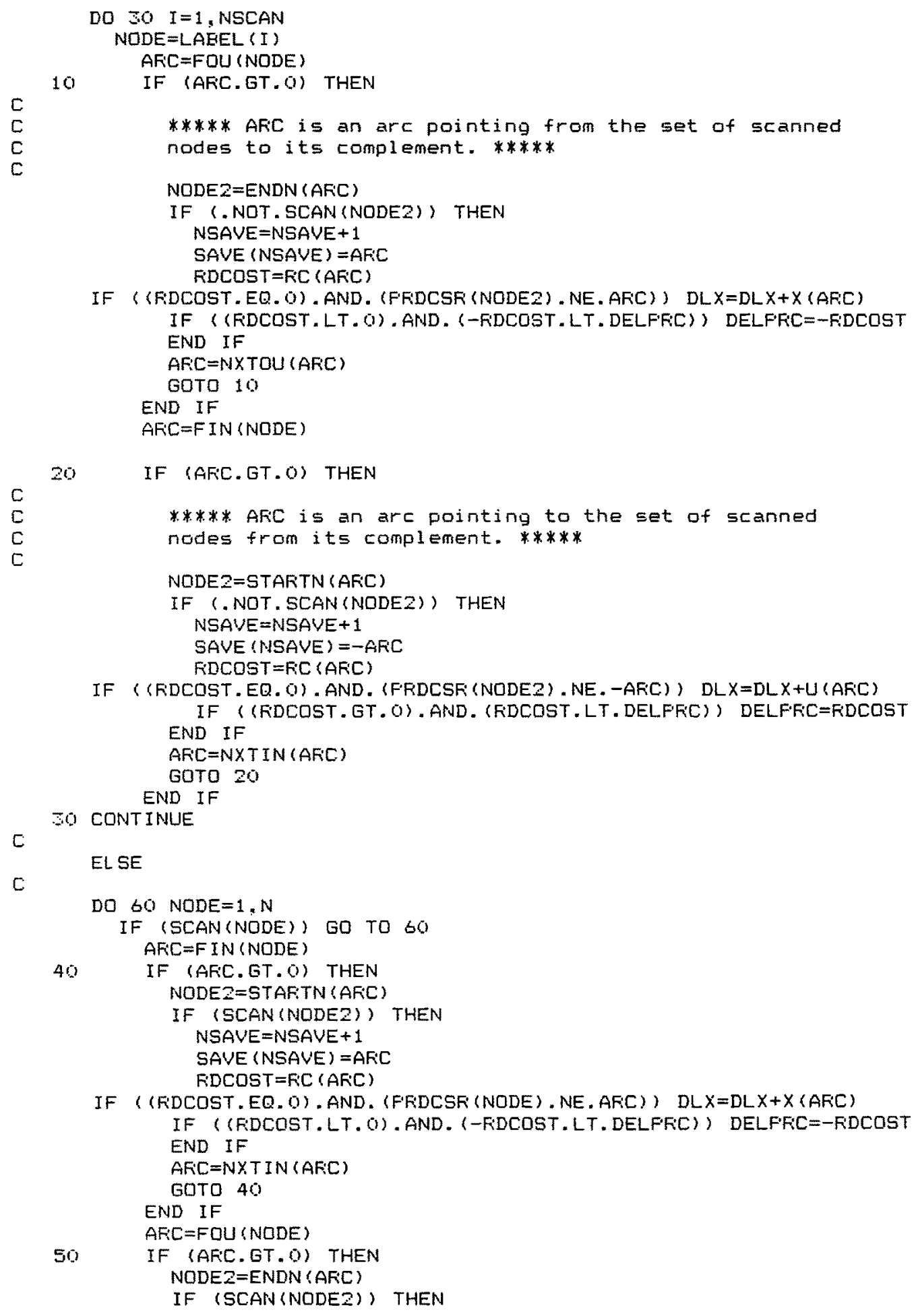




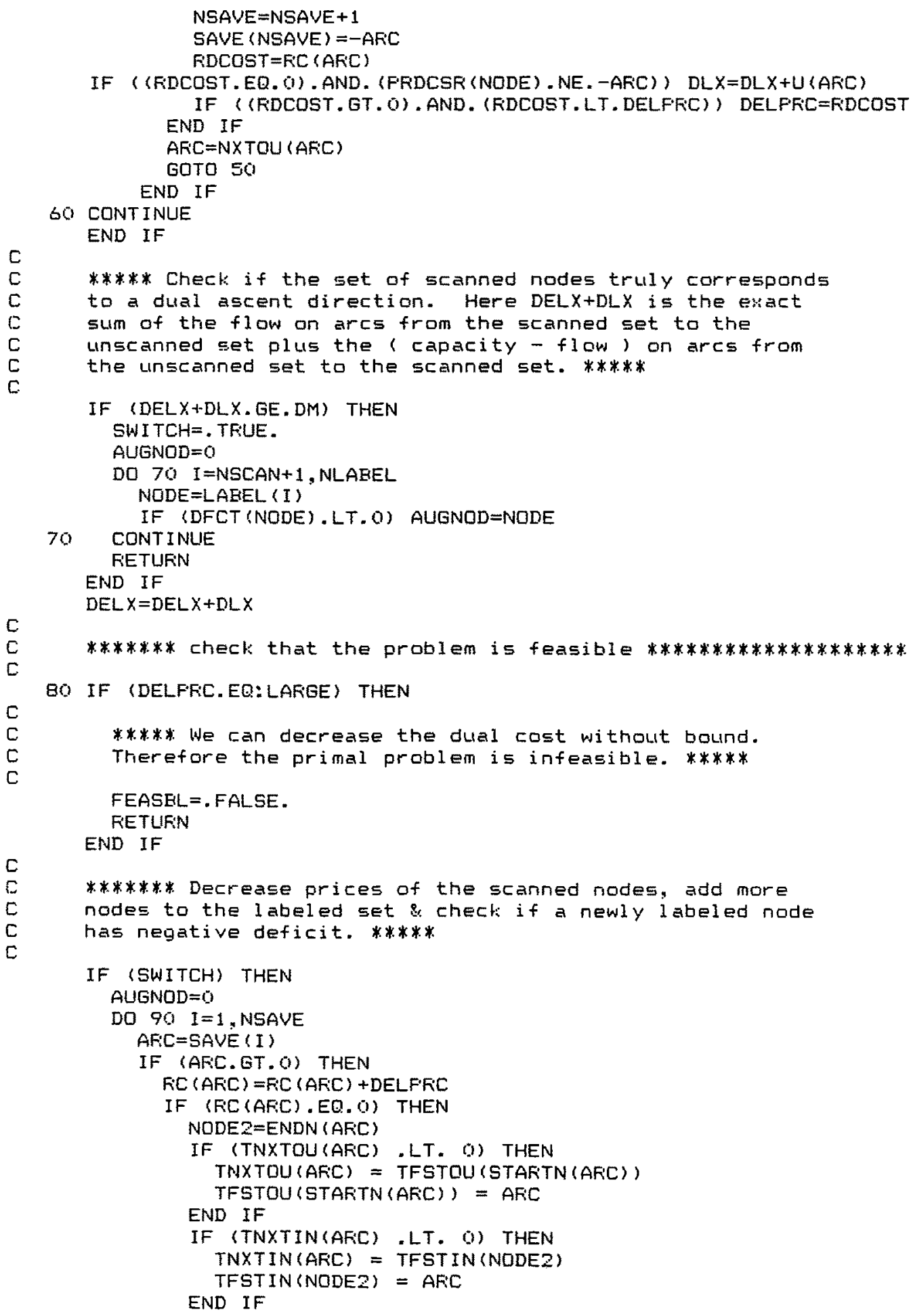




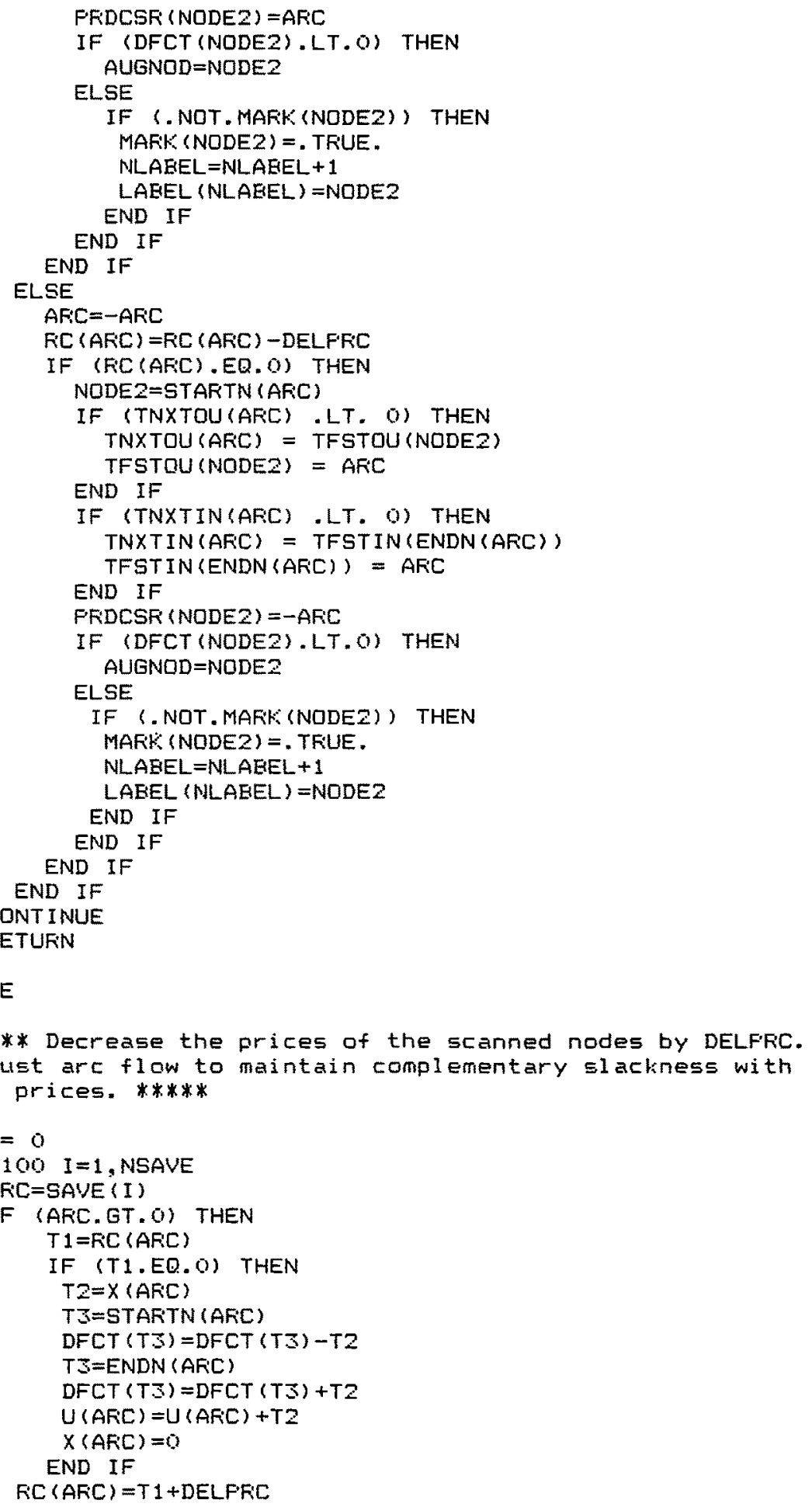




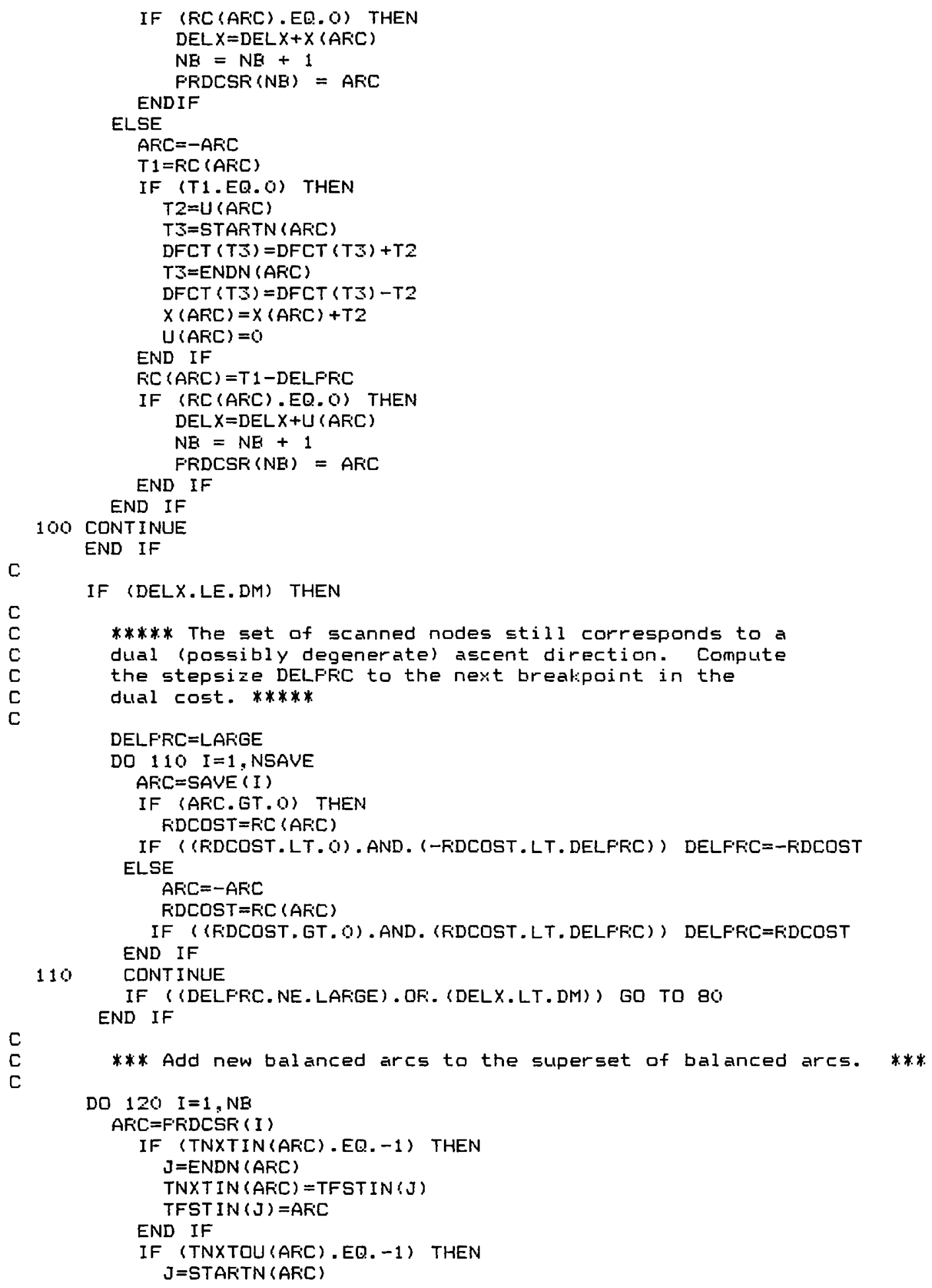




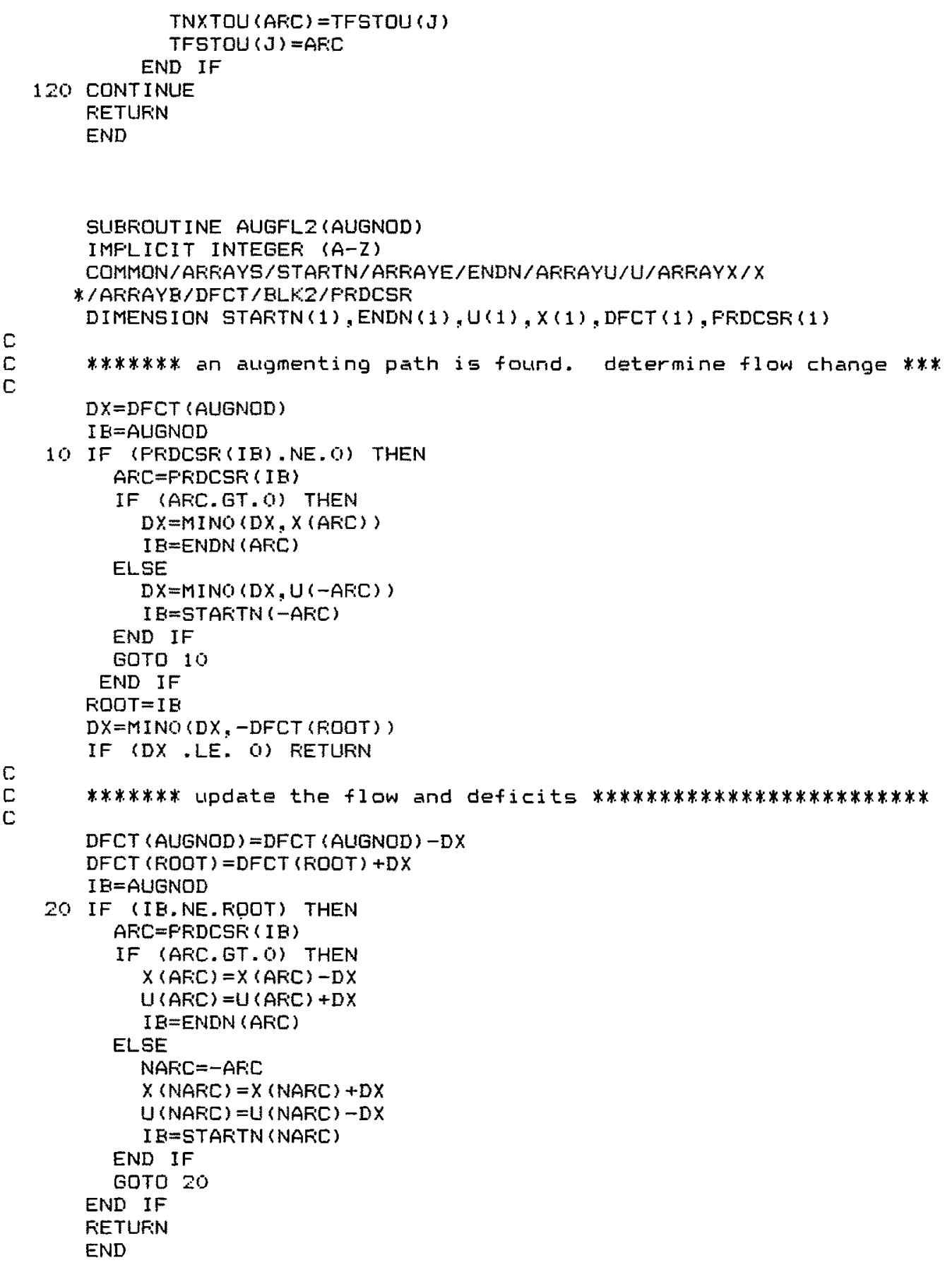

SUEROUTINE ASCNT2 (DM, DELX, NLAEEL, AUGNOD, FEASEL, SWITCH, *NSCAN) 
C
C
C
C
C
C

IMFLICIT INTEGER $(A-Z)$

The two "tree"-based ascent routines have a common temporary storage area whose dimension is set below. The maximum conceivable amount needed equals the number of arcs, but this should never actually occur.

LOGICAL SCAN, MAFK, SWITCH, FEASEL, QUIT

COMMON/ARFAYS/STARTN/ARRAYE/ENDN/ARRAYU/U/ARFAYX/X/ARRAYG/RC

*/ARRAYB/DFCT/ELK1/LABEL/BLK2/FRDCSR/ELK3/FOU/BLK4/

*NXTOU/ELKS/FIN/ELKG/NXTIN/ELKT/SAVE/ELKB/SCAN/ELKG/MARK

$* / L / N, N A$, LAFGE

COMMON/ELK1O/TFSTOU/ELK11/TNXTOU/ELK12/TFSTIN/ELK1S/TNXTIN

COMMON /ASCELK/E

DIMENSION TFSTOU (1), TNXTOU(1), TFSTIN(1), TNXTIN(1)

DIMENSION STARTN(1), ENDN (1), U(1), $X(1), \operatorname{RC}(1), \operatorname{DFCT}(1), \operatorname{LAEEL}(1)$

DIMENSION FFDCSF (1), FOU (1), NXTOU (1), FIN(1), NXTIN(1)

DIMENSION SAVE (1), SCAN(1), MAFK. (1)

c

******* augment flows across the cut \& compute price rise *****

DELF'REC=LAFGE

$D L X=0$

NSAVE $=0$

IF (INSCAN.LE.N/Z) THEN

DO $30 \quad I=1$, NSCAN

NODE=LAEEL (I)

10

ARC $=F I N(N D D E)$

IF (ARE. GT. O) THEN

NODE 2=STARTN (ARC)

IF (. NOT. SCAN (NODE2)) THEN

NSAVE=NSAVE+1

$S A V E$ (NSAVE) $=A R C$

FDCOST $=F C$ ( $A F C$ )

IF ( (RDCOST.EQ. O). AND. (FFDCSF (NODE2). NE. AFIC)) DL $X=D L X+X(A F C)$

IF ((FDCOST.LT. O). AND. (-FDCOST.LT. DELFFC) ) DELFFC $=-F D C O S T$

END IF

ARC $=N \times T$ IN $(A F C)$

GOTO 10

END IF

$A F C=F Q U(N D D E)$

20

IF (ARC. GT.O) THEN

NODE2 $=E N D N(A R C)$

IF (.NOT. SCAN (NODEZ)) THEN

NSAVE $=$ NSAVE +1

SAVE (NSAVE) $=-A R C$

RDCOST $=$ FC $(A F C)$

IF ((FDCOST.EQ. O) . AND. (FFDCSF (NODEZ) . NE. -ARC)) DLX $=D L X+U$ (ARC) IF ((KDCOST.GT. O). AND. (RDCOST.LT.DELFRC) ) DELFRC=RDCOST END IF

$A R C=N \times T O U(A R C)$

GDTO 20

30 CONTINUE

END IF

ELSE

DO $60 \mathrm{NODE}=1, \mathrm{~N}$

IF (SCAN(NODE)) GO TO 60 $A F C=F O U$ (NDDE)

40 IF (AFC. GT. O) THEN NODE2 $=$ ENDN $(A R C)$ 


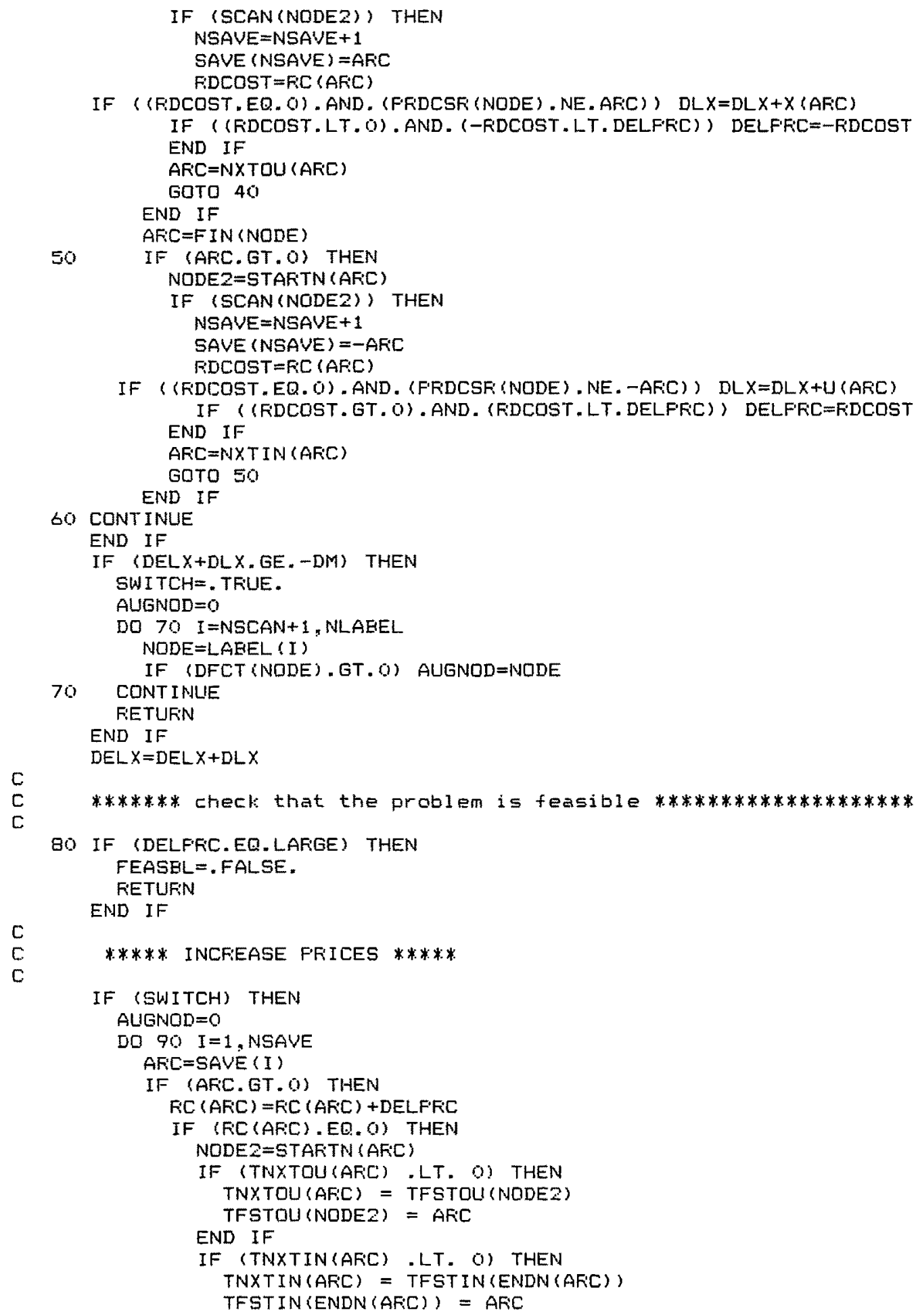




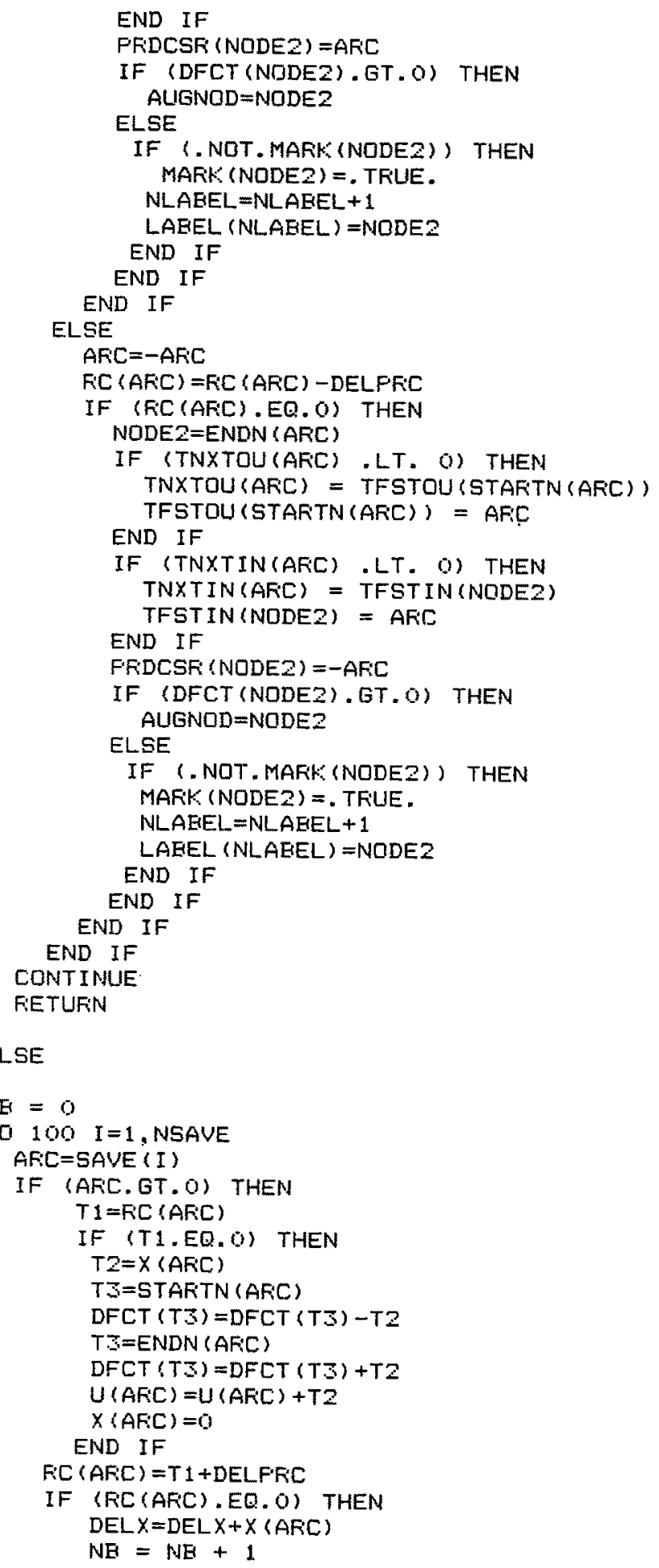




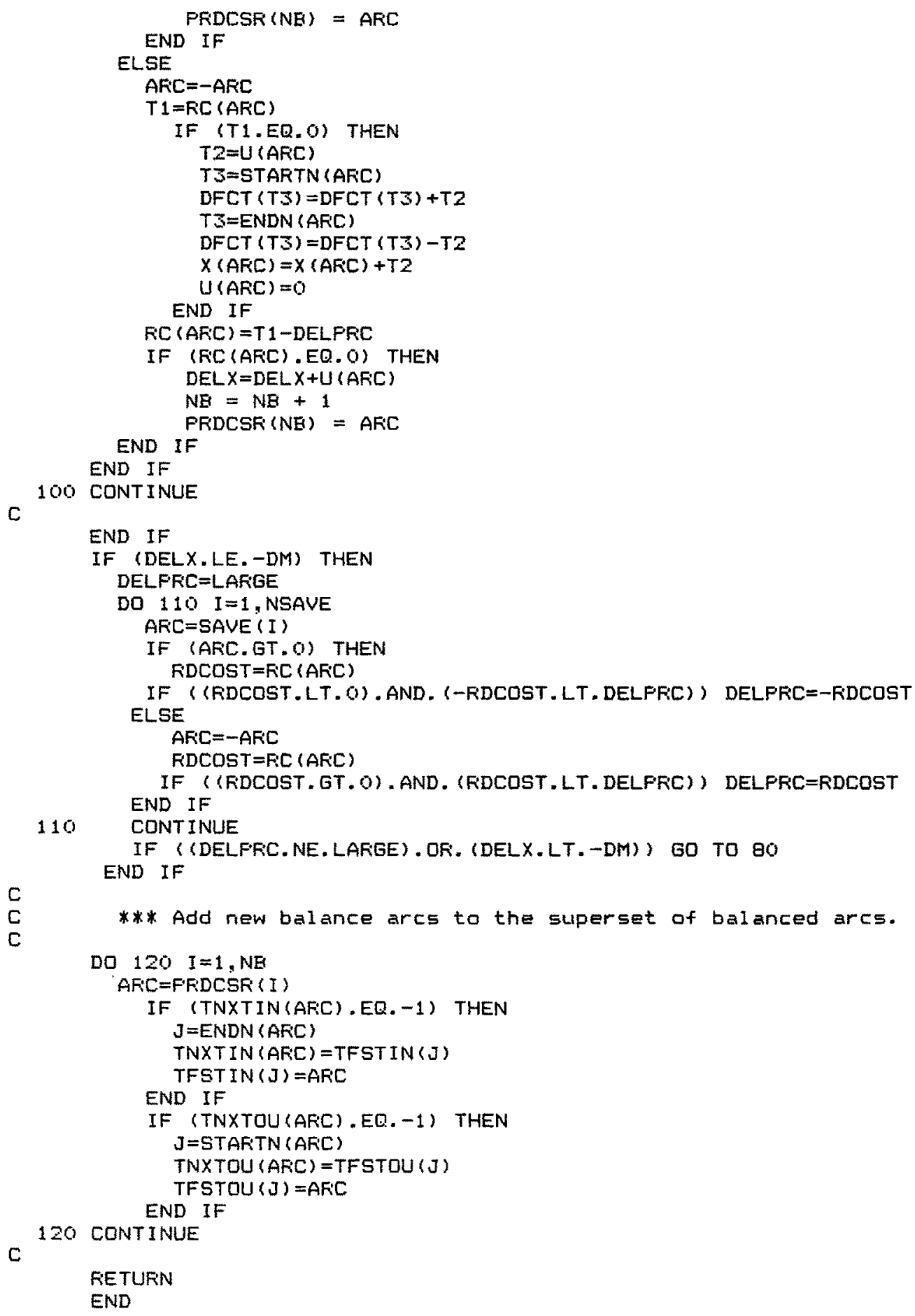




\section{SUBFOUTINE SENSTV}

SENSTTIUTTY ANALYSTS FOR THE MTNTMUM COST NETWORK FLOW FFOELEM.

\begin{tabular}{|c|c|c|}
\hline * $*$ & THE SUEFIOUTINE IS BASED ON THE FAFEF & $* * *$ \\
\hline 来米 & D.P. EEFTSEKAS F F TSENG "THE FELAX CODES FOF: & **** \\
\hline$* * *$ & LINEAF: MINIMUM COST NETMOFE FLOW FFOELEMS", & $* * *$ \\
\hline 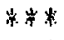 & ANNALS OF OPERATIONS RESEARCH, THIS VOLUME & 事半 \\
\hline$* * *$ & & *⿻丷木 \\
\hline$* * *$ & JN STANDÄFD FOF:TFAN77 & $* * *$ \\
\hline$* * *$ & & 为米。 \\
\hline$* * *$ & DUESTIDNS AND COFNENTS SHOULO EE DIEECTED & $* * *$ \\
\hline 象吢 & DIMITFII BEFTSEEKAG AND FALLL TSENG & $* * *$ \\
\hline$* * *$ & DEPAFTMENT OF ELECTFICAL ENGINEEFING & $* * *$ \\
\hline 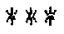 & COMF'UTEF: SEJENCE & 必米 \\
\hline $4 *$ & IABOFATOFY FOF 1MFGFIATIOM AMD DECISJON SYSTEMS & 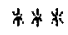 \\
\hline * & M. I.T" CAMEFIDGE "MASSACHUSETTS, M21:9. U.S.A. & $* * *$ \\
\hline
\end{tabular}

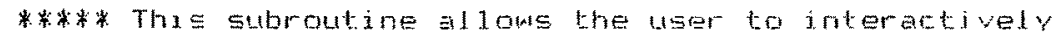
Either change nodal supply* or change flow upper bound of an existing are, or whange cost or an existing are. or delete an existing arc; or add an arc.

NOTE : If in the syekem on whith this subroutine is ran. the varjable local to a subroutine $i s$ re-initialized fo come default value) each time the subroutine is called. bhen the user must male the foldowing currently lacal variables DELAFC, DAFC, DU, ADDAFC, AAFr global by ejther putting them in a comon block or passume them through the ca] 1 ing pincimeter'.

IMFI_ICIT INTEGEF: (A-Z)

CDMMON/AFFAYS/STAFTN/AFFIAYE/ENDN/AFF:AYU/U/AFFAYX/X/AFFAYG/FE:

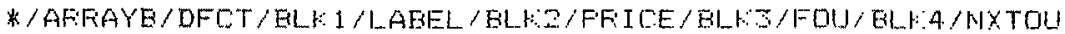

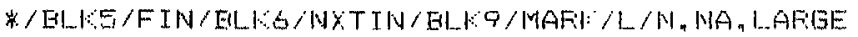

CONION/AFFAYC /C/ELKCAF/CAF/ELFF/FEFEAT

INTEGEF $\operatorname{CAF}(1), U(1), x, 1), \mathrm{C}(1), \mathrm{FC}(1), \mathrm{DFCT}, 1)$

INTEGEF STAFTN(1), ENDN (1) , LAEEL (1) FFICE (1), FOU (1), NXTOU(1),

*FIN(1), NXTTN(1)

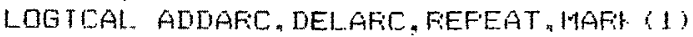

IF $($ NOT. FIEFEAT) THEN

$c$
$c$
$c$
$c$
$c$

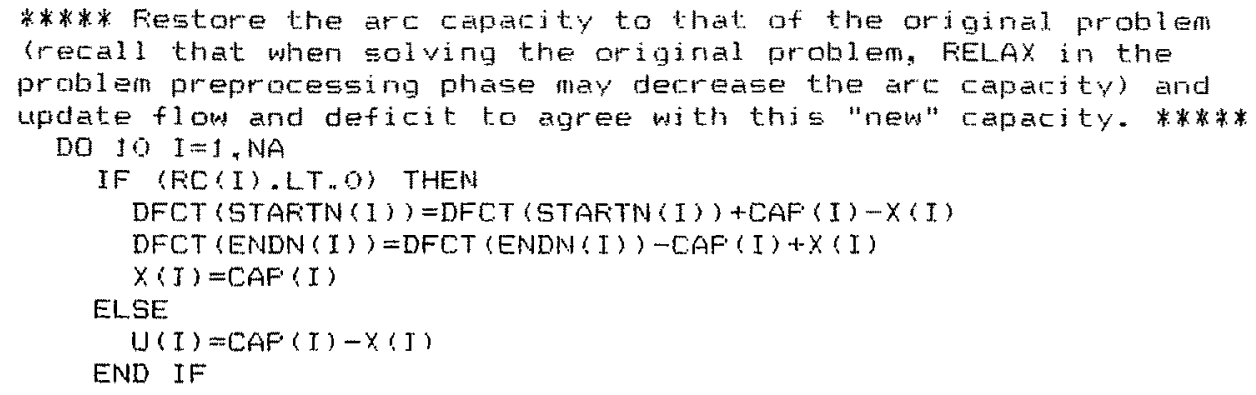


10 $E$

CONT INUE

FIEF'EAT $=$. TFLUE.

END IF

WF: I TE $(6,30)$

WFI I TE $(6,40)$

(WFI ITE $(6,50)$

WR IT TE $(6,60)$

WFI I TE $(6,70)$

WFI TE (6, Q0O)

IF (ADDAF:C) WJ:ITE $(6,90)$ AAF:C

IF (DELAFIC) WFITE (6,100) DAFIC

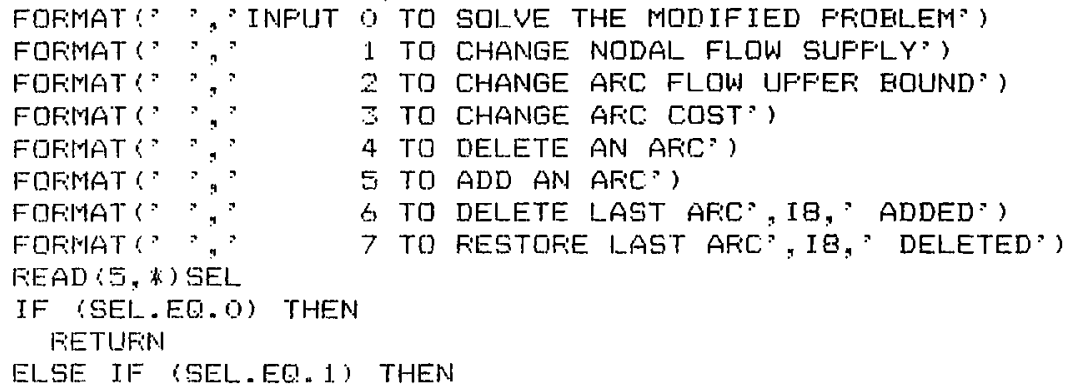




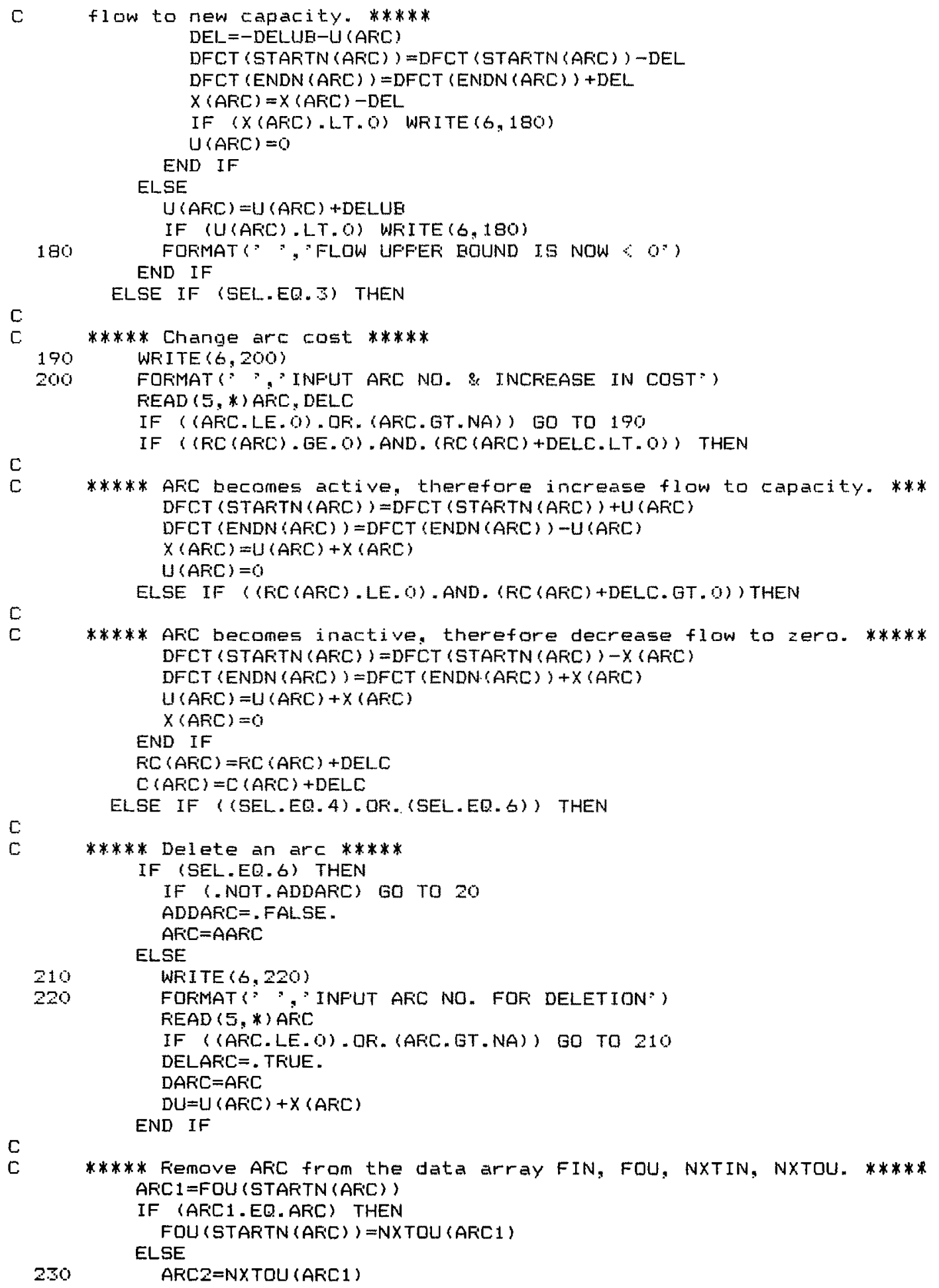




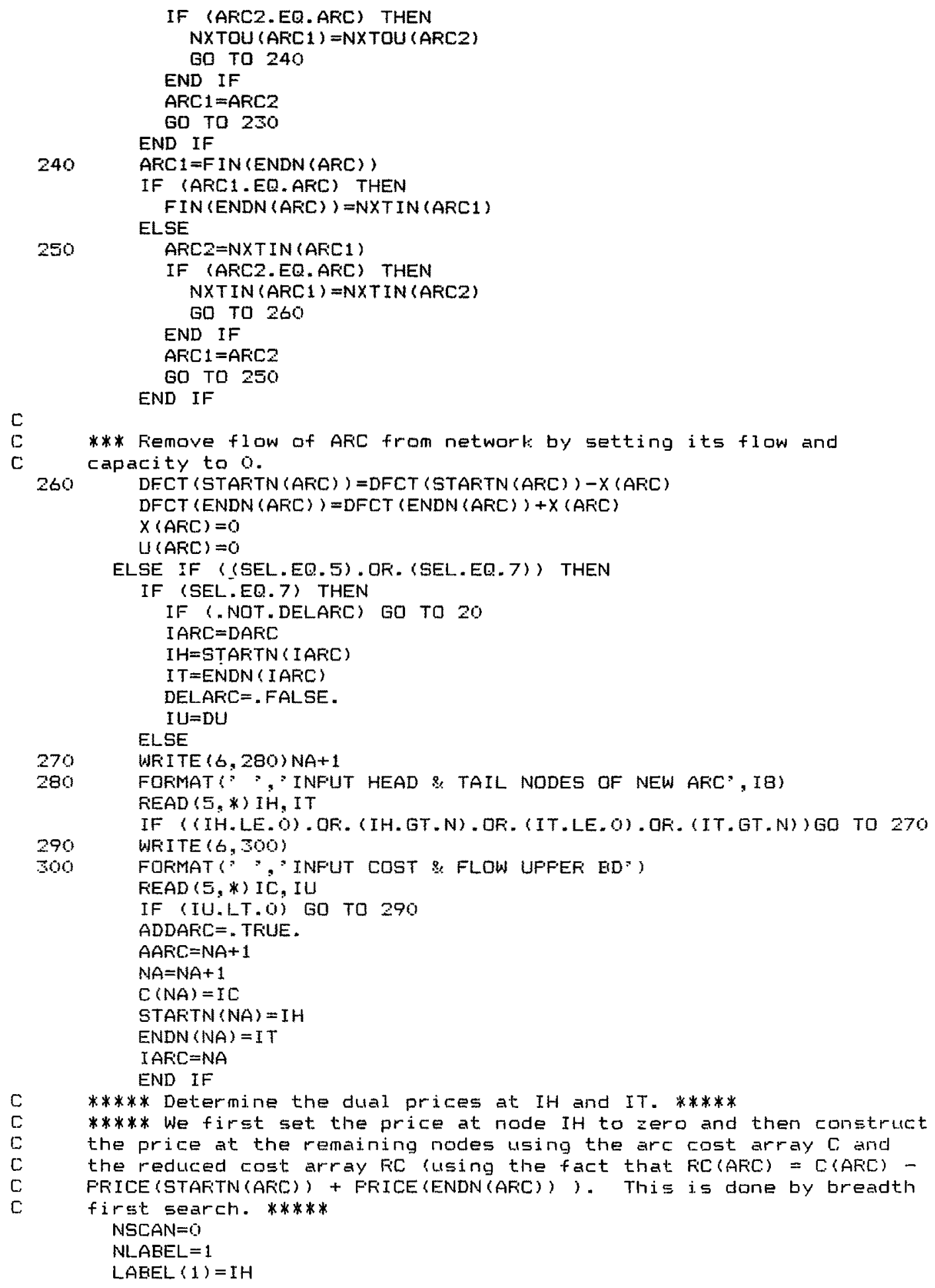




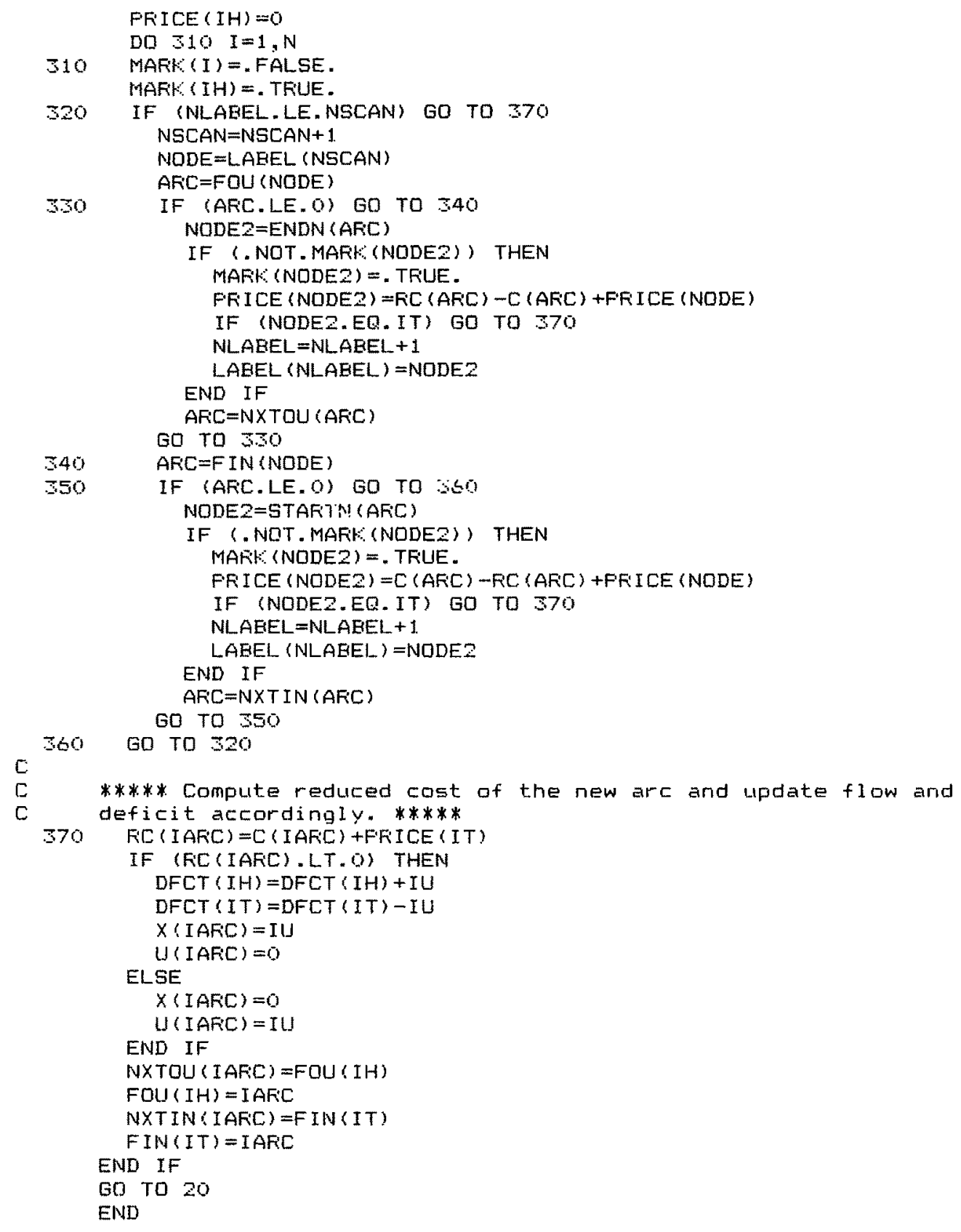

